\title{
SpoVG is an important regulator of sporulation and affects biofilm formation by regulating SpoOA transcription in Bacillus cereus 0-9
}

Qiubin Huang ${ }^{1,2}$, Zhen Zhang ${ }^{1}$, Qing Liu ${ }^{1}$, Fengying Liu ${ }^{1,2}$, Yupeng Liu ${ }^{1,2}$, Juanmei Zhang ${ }^{2,3^{*}}$ and Gang Wang ${ }^{1,2^{*}}$

\begin{abstract}
Background: Bacillus cereus 0-9, a Gram-positive, endospore-forming bacterium isolated from healthy wheat roots in our previous research, is considered to be an effective biocontrol strain against several soil-borne plant diseases. SpoVG, a regulator that is broadly conserved among many Gram-positive bacteria, may help this organism coordinate environmental growth and virulence to survive. This study aimed to explore the multiple functions of SpoVG in B. cereus 0-9.

Methods: The gene knockout strains were constructed by homologous recombination, and the sporulation process of $B$. cereus 0-9 and its mutants were observed by fluorescence staining method. We further determined the spore yields and biofilm formation abilities of test strains. Transcriptional fusion strains were constructed by overlapping PCR technique, and the promoter activity of the target gene was detected by measuring its fluorescence intensity. The biofilm production and colonial morphology of B. cereus 0-9 and its mutants were determined to study the functions of the target genes, and the transcription level of the target gene was determined by qRT-PCR.

Results: According to observation of the sporulation process of B. cereus 0-9 in germination medium, SpoVG is crucial for regulating sporulation stage $V$ of $B$. cereus 0-9, which is identical to that of Bacillus subtilis but differs from that of Bacillus anthracis. In addition, SpoVG could influence biofilm formation of B. cereus 0-9. The transcription levels of two genes closely related to biofilm-formation, sipW and calY, were downregulated in a $\triangle$ spoVG mutant. The role of SpoVG in regulating biofilm formation was further explored by deleting the genes abrB and $\sin R$ in the $\Delta$ spoVG mutant, respectively, generating the double mutant strains $\Delta \operatorname{spoVG} \Delta a b r B$ and $\Delta s p o V G \Delta \sin R$. The phenotypes of these double mutants were congruent with those of the single abrB and $\sin R$ deletion strains, respectively, which showed increased biofilm formation. This indicated that spoVG was located upstream of abrB and $\sin R$ in the regulatory pathway of $B$. cereus biofilm formation. Further, the results of qRT-PCR and the luminescence intensity of transcriptional fusion strains indicated that spoVG gene deletion could inhibit the transcription of SpoOA.
\end{abstract}

\footnotetext{
* Correspondence: Zhangjm@henu.edu.cn; gwangbio@henu.edu.cn

${ }^{2}$ Engineering Research Center for Applied Microbiology of Henan Province, Kaifeng 475004, China

${ }^{1}$ Institute of Microbial Engineering, Laboratory of Bioresource and Applied

Microbiology, School of Life Sciences, Henan University, Jinming Street,

Kaifeng 475004, China

Full list of author information is available at the end of the article
}

(C) The Author(s). 2021 Open Access This article is licensed under a Creative Commons Attribution 4.0 International License, which permits use, sharing, adaptation, distribution and reproduction in any medium or format, as long as you give appropriate credit to the original author(s) and the source, provide a link to the Creative Commons licence, and indicate if changes were made. The images or other third party material in this article are included in the article's Creative Commons licence, unless indicated otherwise in a credit line to the material. If material is not included in the article's Creative Commons licence and your intended use is not permitted by statutory regulation or exceeds the permitted use, you will need to obtain permission directly from the copyright holder. To view a copy of this licence, visit http://creativecommons.org/licenses/by/4.0/ The Creative Commons Public Domain Dedication waiver (http://creativecommons.org/publicdomain/zero/1.0/) applies to the data made available in this article, unless otherwise stated in a credit line to the data. 
Conclusions: SpoVG, an important regulator in the sporulation of B. cereus, is located upstream of Spo0A and participates in regulation of biofilm formation of $B$. cereus 0-9 through regulating the transcription level of spo0A. Sporulation and biofilm formation are crucial mechanisms by which bacteria respond to adverse conditions. SpoVG is therefore an important regulator of SpoOA and is crucial for both sporulation and biofilm formation of B. cereus 0-9. This study provides a new insight into the regulatory mechanism of environmental adaptation in bacteria and a foundation for future studies on biofilm formation of $B$. cereus.

Keywords: Biofilm, SpoVG, Sporulation, Sinl/R, Spo0A, Bacillus cereus

\section{Introduction}

Bacteria have developed various mechanisms, some not yet known, to adapt and respond to adverse environmental factors during the long course of evolution. Biofilm formation and sporulation are two different effective survival strategies that enable bacteria to utilize a greater variety of nutrients, endure rapid environmental changes, and resist multiple adverse threats [1]. For Bacillus cereus, sporulation or biofilm formation is an effective self-protective behavior used to survive environmental pressures, such as nutrient deficiency, high temperature, high salt, antibiotics, etc. $B$. cereus produces a variety of biofilms that differ in their architecture and mechanism of formation, possibly reflecting an adaptation to various adverse environments [2]. B. cereus can also form dormant spores under extremely adverse conditions, germinating when external environmental conditions are permissible for growth.

In Bacillus subtilis, sporulation is a developmental pathway in which sequential, compartmentalized gene expression is achieved by interlocking cascades of regulatory factors and morphological cues [3]. Sporulation of $B$. subtilis has been divided into so-called "stages" 0 to VII using electron microscopy. In the $B$. cereus group (which includes Bacillus anthracis), sporulation is comparable to that in B. subtilis, but there are some differences between different strains [4]. For example, spore-coat assembly begins at the mother-cell-proximal pole of the forespore in B. subtilis, whereas coat material first appears on the long axis of the forespore in B. anthracis and B. cereus [5]. The primary environmental signal for initiation of sporulation is nutrient limitation [6], which could also induce characteristics of other adaptive responses, such as slow growth, non-proliferative stationaryphase cells, or biofilm formation.

Biofilm formation requires a complex regulatory pathway that coordinates gene expression with external environmental conditions $[3,7,8]$. In $B$. subtilis, a matrix component operon of tapA-sipW-tasA is required for biofilm formation. TapA is encoded by tapA, in this operon, with sipW encoding a signal peptidase required for the secretion of both TasA and TapA [9]. The control of biofilm formation is dependent on a molecular switch comprising two transcription repressors and two anti-repressors [10]. Central to this regulatory switch is the repressor SinR [11], a DNA binding protein that represses the transcription of the tapA-sipW-tasA operon involved in both production of an exopolysaccharide [12] and the protein component of the matrix. SinR activity is controlled by the relative levels of various antagonists, such as SinI [13], which binds to $\operatorname{SinR}$ and causes derepression because the SinI-SinR complex is no longer able to bind to DNA $[14,15]$. In $B$. subtilis, biofilm formation can be triggered by the master transcription factor Spo0A, which activates biofilm formation by increasing production of SinI [16]. AbrB was also reported to play a role in the regulation of biofilm formation in B. subtilis. AbrB negatively regulates biofilm formation of $B$. subtilis, which is regulated negatively by Spo0A [17]. In contrast, the regulatory mechanisms that control biofilm formation of $B$. cereus are poorly understood because very few genes involved in biofilm formation have been characterized in this species.

SpoVG was initially identified in B. subtilis and is involved in sporulation via an unknown mechanism [18, 19], although a recent report indicates that SpoVG is a pleiotropic regulatory factor in sporulation [4]. The spoVG gene is highly conserved in bacteria. In B. subtilis, SpoVG negatively regulates asymmetric septum formation and positively regulates cortex formation. In Staphylococcus aureus, which is a nonsporulating bacterium, SpoVG is the main effector molecule of the yabJspoVG operon [3]. It is regulated by $\sigma^{\mathrm{B}}$ and can affect the formation of capsular polysaccharides, regulate the expression of virulence factors, and generate antibiotic resistance [20-22]. In Borrelia burgdorferi, SpoVG is a highly conserved, DNA-binding protein that interacts with cap5, fmtB, exsA, and lukED promoters [23]. According to whole genome sequencing results (GenBank: CP042874.1) and subsequent gene function annotation, the $B$. cereus $0-9$ genome contains a gene whose product is an ortholog of SpoVG (locus_tag: FRY47_00280). 
The amino acid sequence of this SpoVG ortholog was subjected to a blast search on the National Center of Biotechnology Information (NCBI) website and was found to share $90.72 \%$ homology with that of B. subtilis $(\mathrm{SpoVG} \mathrm{168})$, and $100 \%$ sequence identity to SpoVG of $B$. anthracis $\left(\mathrm{SpoVG}_{\mathrm{a}}\right)$. Consequently, the orthologous protein of SpoVG is considered a product of spoVG in B. cereus $0-9$. However, it has not been reported whether biofilm formation of $B$. cereus can be regulated by SpoVG, and how SpoVG participates in responding to environmental stresses. This study explored the role of SpoVG in the environmental adaptation of B. cereus by investigating SpoVG involvement in regulation of sporulation and biofilm formation of the biocontrol strain $B$. cereus $0-9$.

\section{Materials and methods}

\section{Strains, plasmids, culture media, and growth conditions} The properties and culture conditions of B. cereus 0-9 were reported in our previous study [24]. Escherichia coli 116 (pir+) used for the propagation of plasmids was incubated in Lysogeny Broth (LB) at $37^{\circ} \mathrm{C}$ overnight. The recombinant plasmid pMADchi was used in the allelic exchange method for generating complementary mutants [25]. All strains and plasmids used in this study are listed in Table 1. Spore yield was measured in modified germination medium (MG) comprising $1 \mathrm{~g}$ glucose, $7 \mathrm{~g}$ $\mathrm{K}_{2} \mathrm{HPO}_{4}, \quad 3 \mathrm{~g} \quad \mathrm{KH}_{2} \mathrm{PO}_{4}, \quad 0.2 \mathrm{~g}$ yeast extract, $0.1 \mathrm{~g}$ $\mathrm{MgSO}_{4} \cdot 7 \mathrm{H}_{2} \mathrm{O}, 0.01 \mathrm{~g} \mathrm{CaCl}_{2}, 0.001 \mathrm{~g} \mathrm{FeSO}_{4}, 0.1 \mathrm{~g} \mathrm{NaCl}$ and distilled water to $1 \mathrm{~L}$. For biofilm formation, tested strains were cultured in modified Minimal salts glycerol glautamate (MSgg) medium comprising $100 \mathrm{mM} 3-(\mathrm{N}-$ morpholine) propyl sulfonic acid, $2 \mathrm{mM} \mathrm{MgCl}_{2}, 700 \mu \mathrm{M}$ $\mathrm{CaCl}_{2}, 50 \mu \mathrm{M} \mathrm{FeCl}, 50 \mu \mathrm{M} \mathrm{MnCl}_{2}, 5 \mathrm{mM}$ phosphate buffer, $2 \mu \mathrm{M}$ vitamin $\mathrm{B}_{1}, 1 \mu \mathrm{M} \mathrm{ZnCl}_{2}, 5 \mathrm{~g} / \mathrm{L}$ yeast powder, and $5 \mathrm{~g} / \mathrm{L}$ glycerol. MSgg solid media, made by adding $2 \%$ agar powder, was used to observe colony morphology of the tested strains. Nutrient agar (NA) plate was made of $10 \mathrm{~g}$ peptone, $5 \mathrm{~g}$ yeast extracts, $5 \mathrm{~g}$ $\mathrm{NaCl}, 20 \mathrm{~g}$ agar and distilled water to $1 \mathrm{~L}$.

\section{Sequence data analysis and genetic distance calculation}

Whole-genome sequencing (GenBank: CP042874.1) and subsequent gene function annotation revealed that the B. cereus 0-9 genome encodes a SpoVG protein (Protein ID: QEF19539.1). The putative amino acid sequences used in this study were downloaded from the GenBank nucleotide sequence database. Sequence alignment of SpoVG proteins was performed in the DNAMAN program (version 5.2.2.0). BLAST (version 2.10.1) was used to search the GenBank database for SpoVG homologues in other species of bacteria and their amino acid sequences in fasta format were downloaded and inputted into DNAMAN software for multiple sequence alignment.

\section{Construction of gene deletion strains and complemented strains}

The spoVG-knockout mutant strain $\triangle$ spoVG of B. cereus 0-9 (HQ1021) was constructed by a previously described allelic exchange method [26]. In detail, two fragments suitable for allelic exchange were created by cloning two BamHI-XhoI DNA fragments containing locus-specific flanking regions into the $B a m H I$ site of the pMAD vector. These fragments were generated via PCR using the primers in Table S1. Ligation of the two fragments led to precise deletion of the respective open reading frame from the start to the stop codons and to generation of an XhoI site at the locus site. The same procedure was used to create $\Delta \operatorname{sinI}, \Delta \sin R, \triangle a b r B$,

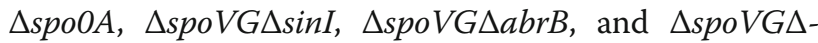
spo0A mutants of $B$. cereus 0-9. A pMADchi vector was constructed using the plasmid pMAD with a chi gene from B. cereus $0-9$, and was used to construct complementation strains [25]. Briefly, after PCR amplification and retrieval, the target gene fragments were digested and inserted into the recombinant pMADchi vector, which was then used to generate the complemented mutant strain by allelic exchange. All strains constructed in this study are shown in Table 1.

\section{Determination of spore yield}

The spore ratios of B. cereus 0-9 and its mutants were determined using the previously reported method of Zhang et al. [24] In brief, each strain cultured in $50 \mathrm{~mL}$ MG medium at $30{ }^{\circ} \mathrm{C}$ and $220 \mathrm{rpm}$ to exponential phase $\left(\mathrm{OD}_{600}\right.$ up to 0.8$)$, and samples collected every 2 hours. One milliliter of each sample suspension was taken, where $0.5 \mathrm{~mL}$ was used to determine the count of spore yield and the other $0.5 \mathrm{~mL}$ was immediately heated at $80^{\circ} \mathrm{C}$ for $10 \mathrm{~min}$. Following incubation, the cultures were spread on LB plates without dilution, and incubated overnight at $30^{\circ} \mathrm{C}$. The following morning, colonies were counted. The sporulation state of each sample was observed under a microscope after staining spores using the classical Schaeffer-Fulton staining method [25]. The sporulation stage in which SpoVG played a key role was determined using two fluorescent dyes to stain the cell membranes and spores, respectively. 4',6-Diamidino-2phenylindole (DAPI) binds specifically to DNA and stains the nucleus with blue fluorescence; FM4-64 binds to neutral cell membranes and appears as red fluorescence under a fluorescence microscope.

\section{Construction of transcriptional fusion strains}

The transcriptional fusion vector was constructed using the plasmid pMADchi. Appropriate primers were 
Table 1 Tested strains and plasmids used in this experiment

\begin{tabular}{|c|c|c|}
\hline Name & Properties and Application & Source \\
\hline \multicolumn{3}{|l|}{ Strains } \\
\hline B. cereus 0-9 & Wild type strain in this study & $\begin{array}{l}\text { Kept in our laboratory, isolated from } \\
\text { wheat root. }\end{array}$ \\
\hline E. coli 116 (pirt) & Plasmid propagation & Purchased from BioVector NTCC \\
\hline $\begin{array}{l}\text { E. coli GM2163 } \\
\text { (dam-) }\end{array}$ & Demethylation & Purchased from BioVector NTCC \\
\hline HQ1021 & The spoVG gene defect mutant, $\triangle$ spoVG & Construct in this study \\
\hline ZY1001 & The $a b r B$ gene defect mutant, $\triangle a b r B$ & Construct in this study \\
\hline FPU1061। & The $\sin$ / gene defect mutant, $\Delta \sin /$ & Construct in this study \\
\hline FPU1062R & The $\sin R$ gene defect mutant, $\Delta \sin R$ & Construct in this study \\
\hline ZL1002 & The spoOA gene defect mutant, $\triangle s p o 0 A$ & Construct in this study \\
\hline HQ2021 & The double knockout strain of spoVG and $\sin /$ genes, $\Delta s p o V G \Delta \sin /$ & Construct in this study \\
\hline HQ2022 & The double knockout strain of spoVG and $\sin R$ genes, $\triangle \operatorname{spoVG} \Delta \sin R$ & Construct in this study \\
\hline HQ2023 & The double knockout strain of spoVG and $a b r B$ genes, $\triangle$ spoVG $\triangle a b r B$ & Construct in this study \\
\hline HQ2024 & The double knockout strain of spoVG and spo0A genes, $\triangle$ spoVG $\triangle$ spoOA & Construct in this study \\
\hline HQ7110 & $\begin{array}{l}\text { DspoVG supplemented with its native spoVG gene by allelic exchange, } \Delta \text { spoVG:: } \\
\text { spoVG }\end{array}$ & Construct in this study \\
\hline HQ7170 & $\begin{array}{l}\text { AspoVG supplemented with spoVG gene from B. subtilis } 168 \text { by allelic exchange, } \\
\Delta \text { spoVG::spoVG } 168\end{array}$ & Construct in this study \\
\hline HQ7121 & $\begin{array}{l}\text { HQ1021 supplemented with its native spo0A gene by reverse complementary, } \\
\triangle \text { spoVG/spoOA }\end{array}$ & Construct in this study \\
\hline HQ7221 & $\begin{array}{l}\text { HQ2024 supplemented with its native spo0A gene by reverse complementary, } \\
\triangle \text { spoVG } \triangle \text { spoOA/spo0A }\end{array}$ & Construct in this study \\
\hline HQ7222 & $\begin{array}{l}\text { HQ2024 supplemented with its native spoVG gene by reverse complementary, } \\
\triangle \text { spoVG } \triangle \text { spoOA/spoVG }\end{array}$ & Construct in this study \\
\hline HQg6071 & B. cereus 0-9 with Pexs Y-GUS labeling & Construct in this study \\
\hline HQg6171 & HQ1021 (AspoVG) with PexsY-GUS labeling & Construct in this study \\
\hline HQg6072 & Wild B. cereus 0-9 with PsipW-GFP labeling & Construct in this study \\
\hline HQg6172 & HQ1021 with PsipW-GFP labeling & Construct in this study \\
\hline HQg6073 & Wild B. cereus 0-9 with PcalY-GFP labeling & Construct in this study \\
\hline HQg6173 & HQ1021with PcalY-GFP labeling & Construct in this study \\
\hline HQg6074 & Wild B. cereus 0-9 with PabrB-GFP labeling & Construct in this study \\
\hline HQg6174 & HQ1021 with PabrB-GFP labeling & Construct in this study \\
\hline HQg6175 & Wild B. cereus 0-9 with Psinl-GFP labeling & Construct in this study \\
\hline HQg6175 & HQ1021 with Psinl-GFP labeling & Construct in this study \\
\hline \multicolumn{3}{|l|}{ Plasmid } \\
\hline $\mathrm{pAD}$ & For gene knockout & Takara, Dalian \\
\hline spoVG-pMAD & For gene knockout, $\mathrm{Amp}^{+} ; \mathrm{Erm}^{+}$ & Construct in this study \\
\hline abrB-pMAD & For gene knockout, $\mathrm{Amp}^{+} ; \mathrm{Erm}^{+}$ & Construct in this study \\
\hline pAD-pgal-JT & For reverse complementation & Stored in our laboratory \\
\hline pMAD-chi & For gene complementation, $\mathrm{Cm}^{+}$ & Construct in this study \\
\hline pET28a-gapB & Expression of protein, $\mathrm{Km}^{+}$ & Construct in this study \\
\hline
\end{tabular}

designed based on known gene sequences. The promoter fragment of the exs $Y$ gene and the open reading frame of the gus gene were amplified by PCR, respectively, and used as templates for overlapping PCR amplification to obtain the complete fragment PspoIIR-gus and PexsYgus. After enzyme digestion by EcolRI and XhoI, the PspoIIR-gus or PexsY-gus fragment was inserted into pMADchi at EcolRI and XhoI sites to generate the 
recombinant plasmid PspoIIR-gus-pMADchi and PexsYgus-pMADchi, respectively. The promoter fragment of the $a b r B$ gene and a green fluorescent protein (GFP) fragment were amplified by PCR, respectively, and used as templates for overlapping PCR amplification to obtain the complete fragment PabrB-GFP. The PabrB-GFP fragment was inserted into pMADchi at EcolRI and XhoI restriction enzyme sites to generate the recombinant plasmid PabrB-gfp-pMADchi. The same method was employed to construct transcriptional fusion vectors PsipW-gfp-pMADchi, PcalY-gfp-pMADchi, PsinI-gfppMADchi and PspoOA-gfp-pMADchi. Using the previously described allelic exchange method [9], these transcriptional fusion vectors were transferred into $B$. cereus 0-9 and $\Delta$ spoVG mutants, respectively, to construct the mutant strains with fluorescent label. All mutant strains constructed in this study are shown in Table 1.

GUS protein is a glucuronidase that can react with the substrate 4-methylumbelliferyl beta-D-glucuronide dihydrate (MUG) to produce fluorescent 4methylumbelliferyl (MU). MU content was measured by fluorescence spectrophotometry (Promega GloMax, USA) with an excitation wavelength of $365 \mathrm{~nm}$ and emission wavelength of $465 \mathrm{~nm}$. Measuring fluorescence intensity reflects the expression of the exs $Y$ promoter. Enzyme activity ( $1 \mathrm{U}$ ) of GUS was defined as the change of fluorescence intensity per unit of protein per hour. The fluorescence intensity of GFP protein was measured by fluorescence spectrophotometry (Promega GloMax, USA) with an excitation wavelength of $488 \mathrm{~nm}$ and emission wavelength of $510 \mathrm{~nm}$.

\section{Growth curve measurements}

Growth curves of B. cereus strains in LB and MSgg medium were determined by inoculating a single bacterial colony into $5 \mathrm{~mL} \mathrm{LB}$ medium and culturing overnight at $37^{\circ} \mathrm{C}$ with shaking. One milliliter of overnight culture medium was centrifuged and the collected bacteria were washed three times with sterile water then re-suspended with different volumes of sterile water to ensure the $\mathrm{OD}_{600}$ of the tested strains was consistent. The suspensions were inoculated into the test medium at a ratio of 1:100 and were cultured in a Bioscreen C (Oy Growth Curves $\mathrm{Ab}$ Ltd., Finland) at $37^{\circ} \mathrm{C}$ with 30 min intervals to automatically record the growth curves of the tested strains.

\section{Determination of biofilm formation}

Solid surface-associated biofilm formation was estimated by the crystal violet staining method with some modifications [25]. A single colony of B. cereus $0-9$ and its transformants were inoculated into $5 \mathrm{~mL}$ LB medium and incubated overnight at $30^{\circ} \mathrm{C}$. Approximately $30 \mu \mathrm{L}$ of the overnight culture was inoculated into $3 \mathrm{~mL}$ MSgg medium in glass culture tubes with a diameter of $0.7 \mathrm{~cm}$. The tubes were incubated in an upright position at $30^{\circ} \mathrm{C}$ for 5 days before surface pellicles and cultures were carefully removed from the tubes. The remaining cells and matrices in each tube were stained with $3.5 \mathrm{~mL}$ of $0.1 \%$ $(\mathrm{w} / \mathrm{v})$ crystal violet solution for $20 \mathrm{~min}$ at $25^{\circ} \mathrm{C}$. After washing three times with distilled water, the crystal violet attached to the biofilm was solubilized in $3.5 \mathrm{~mL}$ of $10 \%(\mathrm{w} / \mathrm{v})$ SDS and quantified in $200 \mu \mathrm{L}$ of the solution by measuring the absorbance at $570 \mathrm{~nm}$. The experiment was repeated five times for each strain.

\section{Assays of complex colony formation}

Colony architecture was analyzed using the method of Diethmaier et al. [10] with slight modifications. Strains of $B$. cereus were precultured in $\mathrm{LB}$ to an $\mathrm{OD}_{600}$ of 0.6 to 0.8 , and $1.0 \mathrm{ml}$ of each culture was pelleted and resuspended in $100 \mu \mathrm{L}$ sterile supernatant. Approximately $2 \mu \mathrm{L}$ of each cell suspension was spotted onto an NA plate and incubated at $30^{\circ} \mathrm{C}$ for 2 to 3 days. The overall pictures of colonies were photographed using a digital single-lens reflex camera (Canon EOS 6D, Japan), and the close-up of individual colonies was photographed by stereomicroscope (Leica S9I, Germany).

\section{Real-time quantitative reverse transcription-PCR}

Real-time quantitative PCR (RT-qPCR) was used to determine the transcription levels of sip $W, c a l Y, a b r B$, $\sin I$, and spoOA genes in B. cereus $0-9$ and $\Delta$ spoVG. $B$. cereus $0-9$ and its derivative strains were cultured in MSgg medium at $30^{\circ} \mathrm{C}, 220 \mathrm{rpm}$ for $24 \mathrm{~h}$ and then harvested. Total RNA was extracted as described previously (Gao et al., 2017) and cDNAs were synthesized using a Fast Quant RT Kit (Tiangen Biochemical Technology, Beijing, China) according to the manufacturer's instructions. For PCR, a GoTaq qPCR Master Mix kit (Shanghai Promega, Shanghai, China) was utilized and qRT-PCR was performed on a Cycler instrument (Bio-Rad, California, USA) as per $B$. cereus 0-9 the manufacturer's recommended protocol, using the primers listed in Table S1.

\section{Statistical evaluations}

Vegetative growth curves of the wild-type and mutant strains were generated by plotting the average outcomes $\left(\mathrm{OD}_{600}\right)$ of three experiments for each strain. The differences in biofilm formation were analyzed by one-way analysis of variance (ANOVA) followed by Tukey's pairwise post-hoc comparisons.

\section{Results}

SpoVG regulates the sporulation of $B$. cereus $0-9$

The sporulation process in B. cereus $0-9$ and its $\triangle$ spoVG mutant was observed using classic Schaeffer-Fulton 
staining method [27] following incubation of the strains in MG medium. Deletion of spoVG inhibited sporulation of B. cereus 0-9 (Fig. 1). After culturing for $6 \mathrm{~h}$, wildtype $B$. cereus $0-9$ had formed spore precursors, but the $\Delta$ spoVG mutant had not. After $24 \mathrm{~h}$, B. cereus $0-9$ had released a mass of mature spores, but the mutant did not. Further, we determined the spore yield ratio and found $\triangle$ spoVG mutant could not produce mature spores (Table S2). Our results were quite in accord with recent report of Chen et al. [4] that in Bacillus anthracis, whose spoVG deletion mutant completely lost sporulation ability and it could not form an asymmetric septum. Therefore, it was concluded that SpoVG protein may play an important role in regulating sporulation of $B$. cereus 0 9.

\section{SpoVG regulates spore-coat formation in stage $V$ of $B$. cereus $0-9$ sporulation}

To determine the sporulation stage in which SpoVG plays a key role in B. cereus $0-9$, two distinct fluorescent dyes were used to stain the cell membranes and spores, respectively. The sporulation process in $B$. cereus $0-9$ was revealed by fluorescent staining (Fig. 2). There was no significant difference in the process of cell differentiation between $B$. cereus $0-9$ and $\triangle$ spoVG mutant at the first stages $(\mathrm{I} \sim \mathrm{IV})$ of culture initiation. After cultured for $8 \mathrm{~h}, B$. cereus $0-9$ had entered stage $\mathrm{V}$ because the red pre-spore coat in the mother cell was visible. After $24 \mathrm{~h}$, we can see clearly that the red spore-precursor turned out in stage VI, and just red dots (mature spores) can be seen in stage VII. However, the sporulation process of the $\Delta$ spoVG mutant remained at stage IV, with the formation of pre-spores but no outer membranes. We extended the culture time to $48 \mathrm{~h}$, and $\Delta$ spoVG still stagnated in the stage IV, while mature spores had been released by B. cereus $0-9$ at this time. This indicated that SpoVG was instrumental in sporecoat formation in stage $\mathrm{V}$ of $B$. cereus $0-9$ sporulation. These results are consistent with those of $B$. subtilis, but differ from B. anthracis where SpoVG protein affects asymmetric septum formation in stage II of sporulation [4]. This may be due to differences in gene expression levels and metabolic regulation mechanisms of bacteria under different environmental and culture conditions.

The role of SpoVG in stage V of sporulation (the stage of spore-coat formation) was further investigated by constructing two pairs of transcriptional fusion mutants with PspoIIR-GUS and PexsY-GUS labelling, respectively, in a wild type and spoVG background (Table 1). GUS activities of these strains were determined, which reflects the expression level of the gene spoIIR and exs Y. In the whole cultivation process, the expression levels of spoIIR were not different in the wild-type strain and $\triangle$ spoVG mutant (Fig. S1). Thanks to SpoIIR is a key factor in the

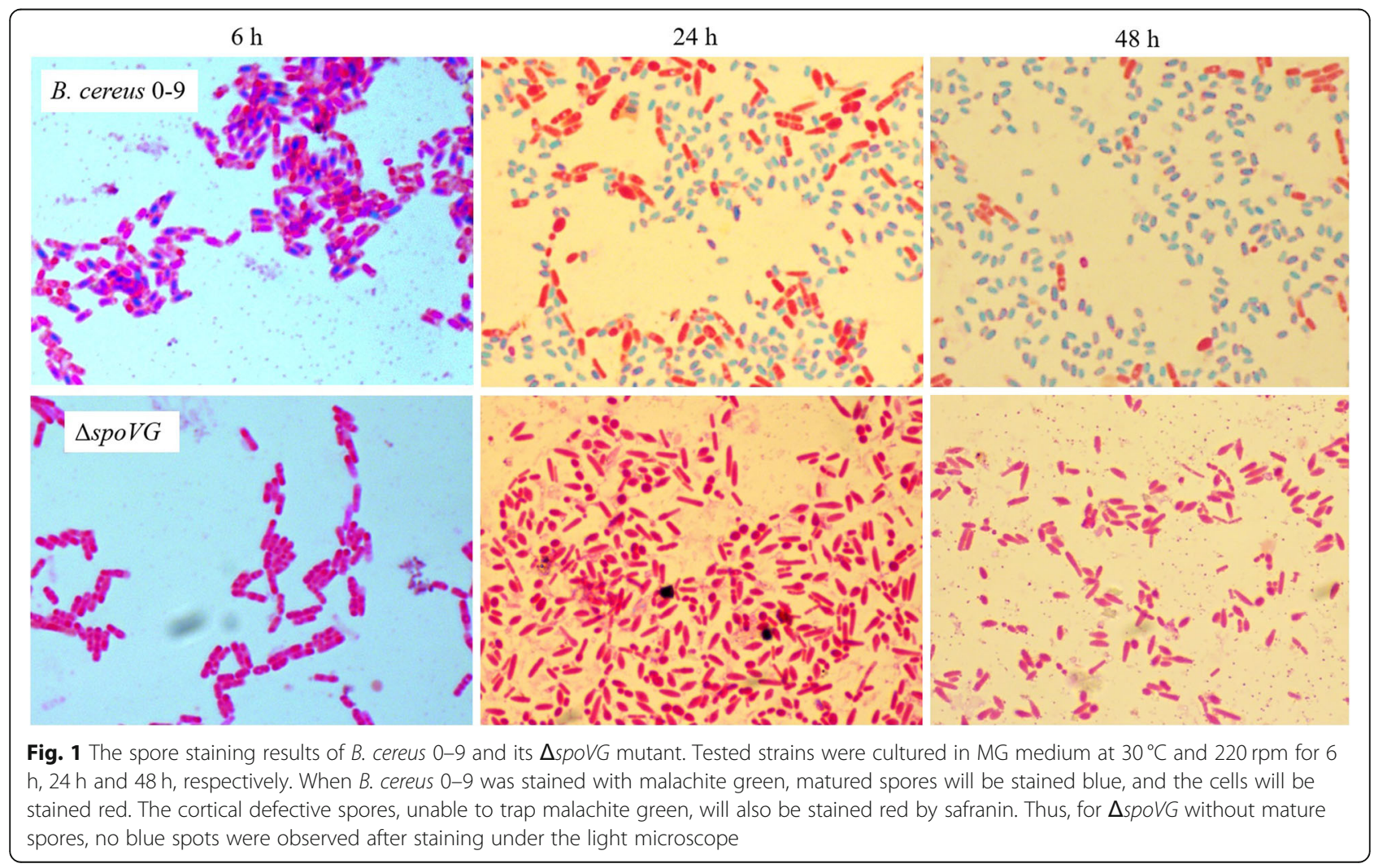




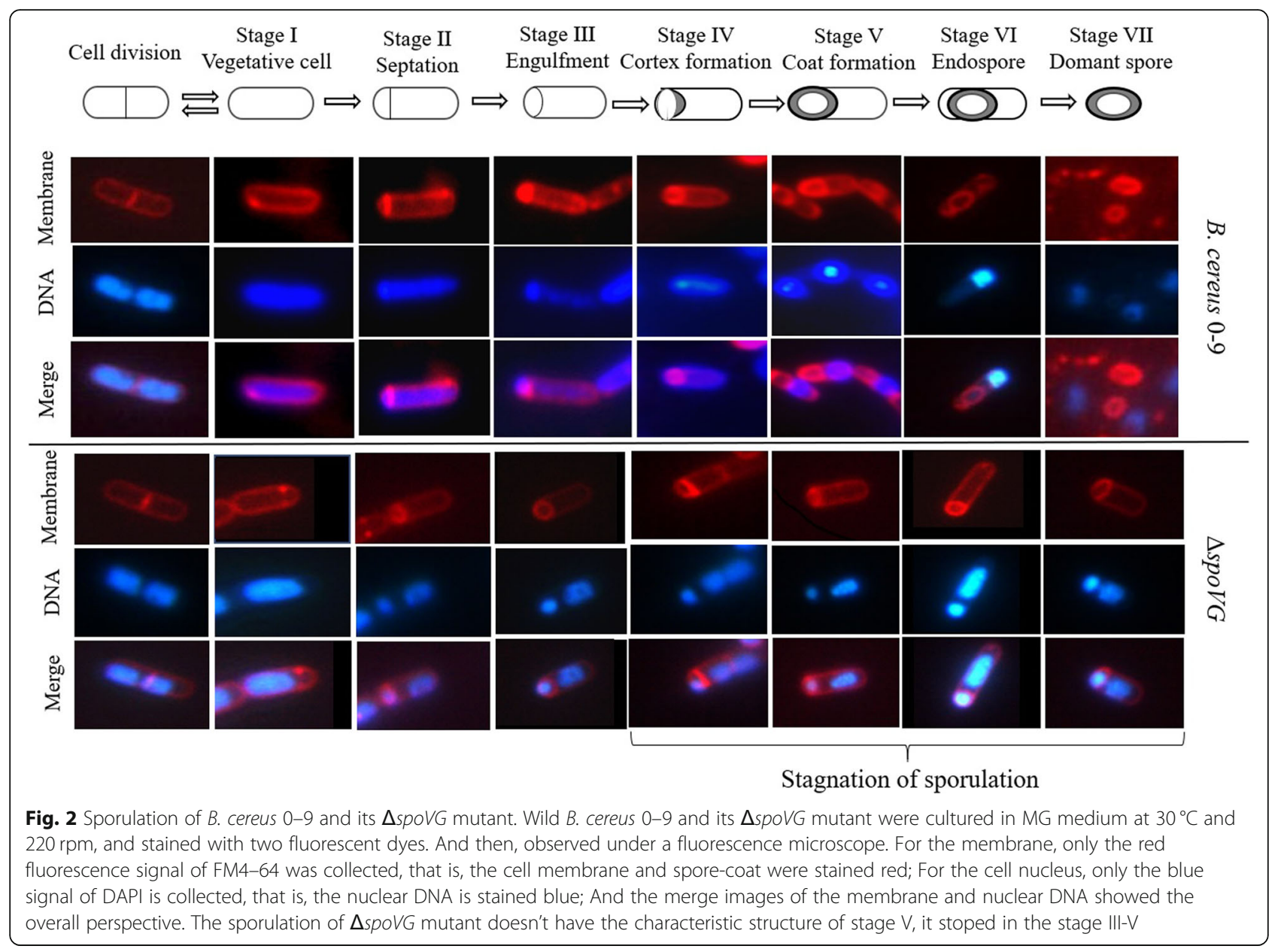

regulation of III stage of sporulation, it indicates that spoVG gene deletion has no effects on the first three stages of sporulation. But for exs $Y$, in the first $5 \mathrm{~h}$, GUS activity was low in both wild type $B$. cereus $0-9$ and $\triangle$ spoVG mutant when they were cultured in MG medium, and there was no significant difference between the wild-type strain and $\Delta$ spoVG (Fig. 3). However, after culture for 6-7 h, GUS activity of B. cereus 0-9 increased by six to seven times, while that of $\triangle s p o V G$ remained unchanged. This indicated that the spoVG gene deletion prevented transcription of exs $Y$. SpoIIR, required for activation of $\sigma^{\mathrm{E}}$ in the mother cell, is a key factor which plays an important role in the sporulation stage III [28]. ExsY is reported to be a key factor that controls formation of the outer membrane of spores [5]. Thus, SpoVG was concluded to have a critical role in spore-coat formation.

\section{SpoVG is involved in regulating biofilm formation of B. cereus 0-9}

The role of SpoVG in biofilm formation of B. cereus 0-9 was also investigated. $B$. cereus $0-9$ could generate biofilm when it was cultured to the post-stabilization period in MSgg medium, but the $\triangle$ spoVG mutant did not form wrinkled biofilm on the gas-liquid interface in MSgg medium (Fig. 4, A). To clarify the relationship between spoVG and biofilm phenotype, two complemented strains of $\Delta$ spoVG::spoVG and $\Delta$ spoVG::spoVG ${ }_{168}$ (Table 1) were constructed using the vector of pMADchispoVG and pMADchi-spoVG $G_{168}$, respectively, which provided the spoVG gene in trans. Complementation of spoVG by the native spoVG gene ( $\triangle$ spoVG::SpoVG) fully recovered the biofilm formation abilities of the mutant, while that of the heterologous complement strain $\Delta$ spoVG::spoVG ${ }_{168}$ was also restored $83.64 \%$. Quantitative analysis showed that the biofilm yield of the $\Delta s p o V G$ mutant decreased $53.0 \%$ compared with that of wildtype $B$. cereus $0-9$, whereas biofilm production by the homologous complemented strains $\Delta$ spoVG::SpoVG was not significantly different to wild-type $B$. cereus $0-9$. Thus, the gene spoVG may be fully reflective of involving in the biofilm formation of B. cereus 0-9.

Bacteria can form another type of biofilm on solid surfaces, which can be fully reflective of the morphological characteristics of bacterial colonies [2, 29]. Therefore, biofilm formation of the $\triangle s p o V G$ 


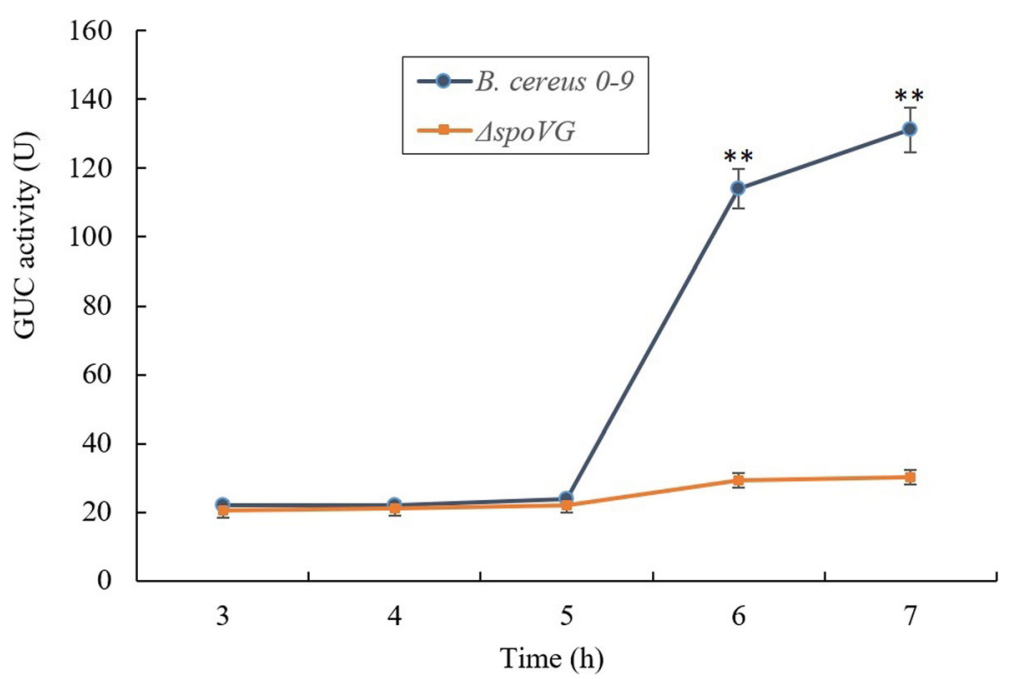

Fig. 3 The enzymatic activities of GUS in wild-type B. cereus 0-9 and $\Delta$ spoVG mutant. Test strains were cultured in MG medium for different culturing times. $1 \mathrm{U}$ of GUS was defined as the change of fluorescence intensity per unit of protein in per hour. Each bar represents mean and standard deviations of the mean of all the 3 measurements. " $* * *$ means $P<0.01$ compared with the data of $\Delta$ spoVG group

mutant strains was observed on MSgg plate media. Compared with the colony morphology of wild-type B. cereus $0-9$, colonies of the $\Delta$ spoVG mutant appeared smooth and the folds on the surface of the wild-type strain completely disappeared in the $\Delta$ spoVG mutant (Fig. 5). Folds were restored in both $\Delta$ spoVG::spoVG and $\Delta$ spoVG::spoVG 168 strains, demonstrating that both native and exogenous spoVG could be used for complementation and restore its phenotype. The folds are a type of biofilm present on solid media and these results were consistent with those obtained by static culture in liquid MSgg medium. This indicated that SpoVG was crucial for biofilm formation of B. cereus 0-9.

\section{SpoVG is involved in regulating both Sinl/R and AbrB systems}

To determine whether SpoVG influenced biofilm formation of $B$. cereus $0-9$ via $\operatorname{SinR}$ or AbrB, two double knockout strains, $\Delta$ spoVG $\Delta \sin R$ and $\Delta s p o V G \Delta a b r B$, were constructed, and their ability to form biofilms on MSgg plate were investigated. The double knockout strain $\triangle$ spoVG $\Delta \sin R$ showed the similar colony morphology as the single knockout strain $\Delta \sin R$ (Fig. 6), that is, the colony surface folds increased meaning the amount of biofilm formation had increased. This indicated that SpoVG is located upstream of $\sin R$ and likely to play a role in regulating $\sin R$ gene expression. Furthermore, the double knockout strain $\triangle$ spoVG $\triangle a b r B$ also showed

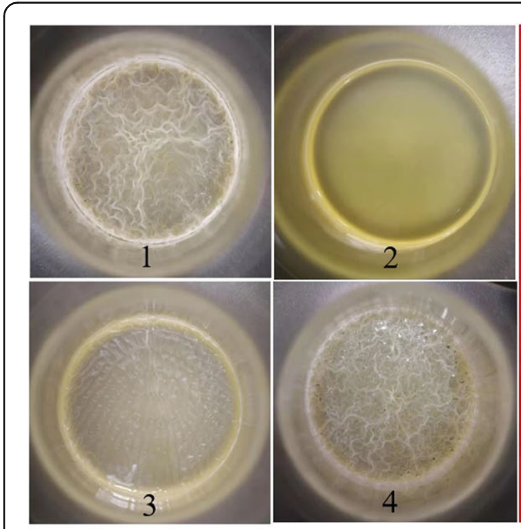

(A)

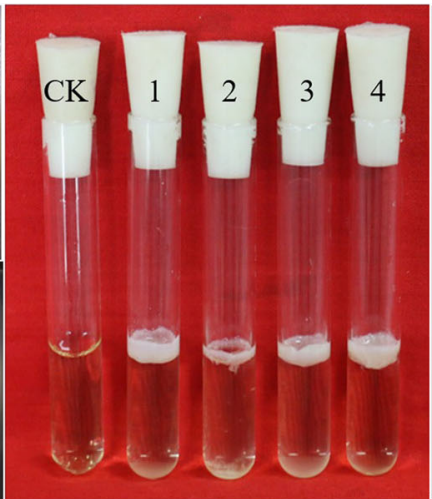

(B)

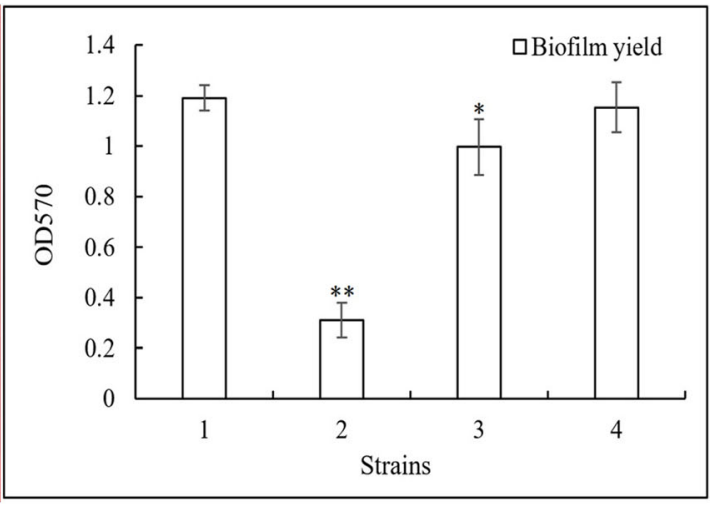

(C)

Fig. 4 Image and yields of biofilms formed by B. cereus 0-9 and its $\Delta$ spoVG mutants. (A) Top view of the biofilms; (B) Side view of the biofilms in test tube; $(C)$ The quantitative determination results of biofilm yields. Each bar represents the mean of all the measurements and its standard deviations; "*" means $0.01<P<0.05$; "**" means $P<0.01$. (1) B. cereus 0-9; (2) $\Delta$ spoVG; (3) $\triangle$ spoVG::spoVG 168 ; (4) $\triangle$ spoVG::spoVG and blank MSgg medium was used for negative control (CK) 


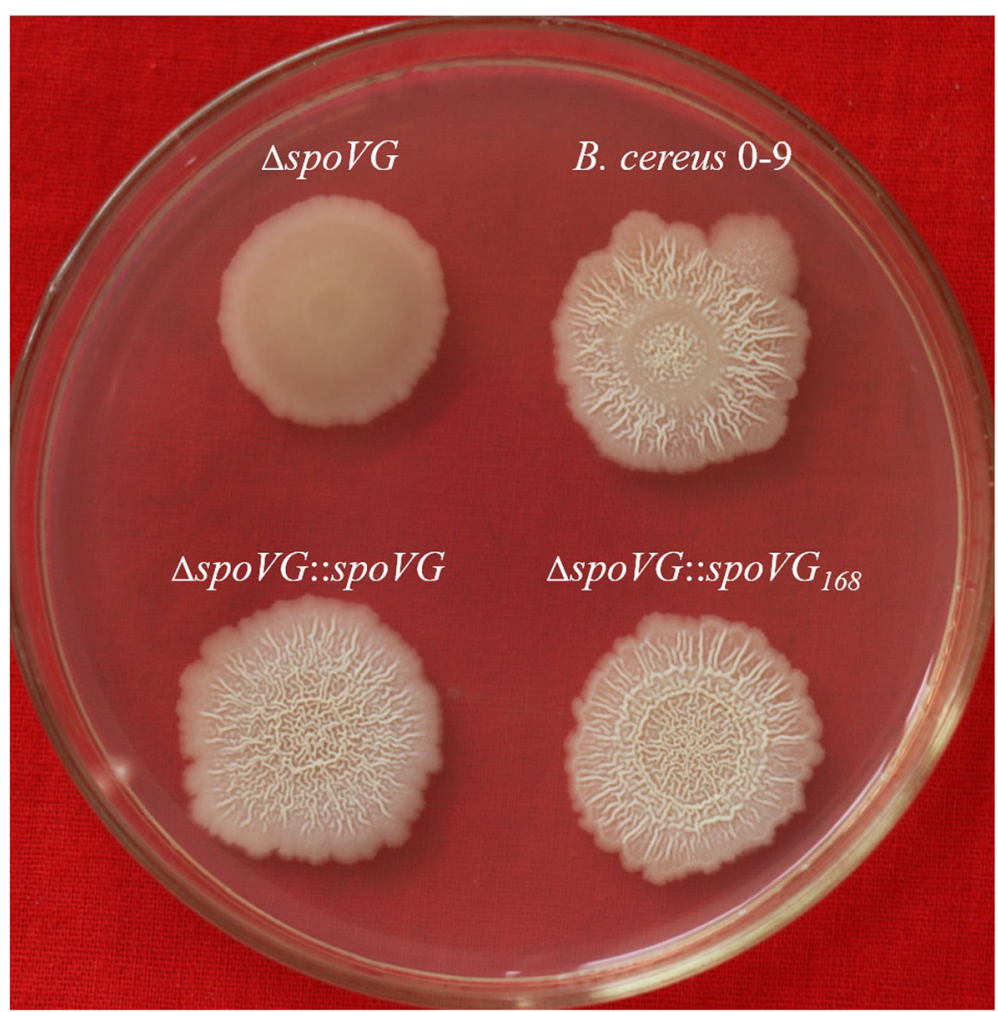

Fig. 5 The colonial morphology of B. cereus 0-9, $\Delta$ spoVG and its complemented mutants. Tested strains were seeded on an NA plate and incubated at $30^{\circ} \mathrm{C}$ for 2 to 3 days. And then, took photos by digital camera. (1) B. cereus 0-9; (2) $\Delta$ spoVG; (3) $\triangle$ spoVG::spoVG; (4) $\triangle$ spoVG::spoVG 168

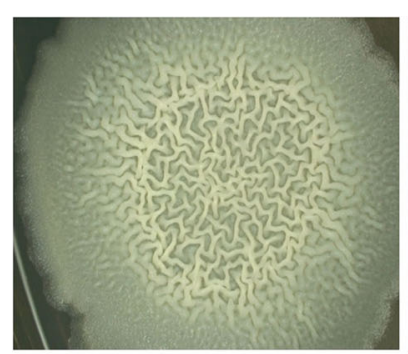

B. cereus $0-9$

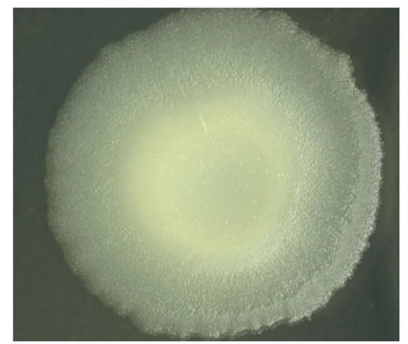

$\triangle \operatorname{spoVG}$

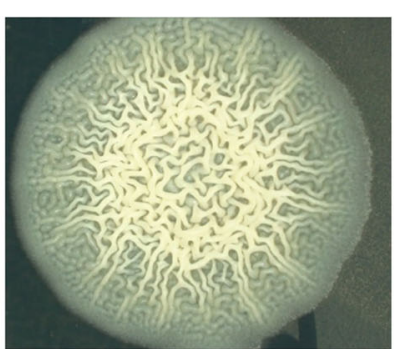

$\Delta \sin R$

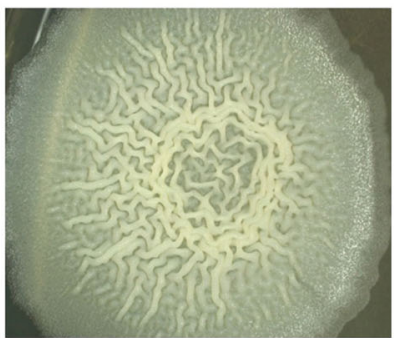

$\Delta s p o V G \Delta \sin R$

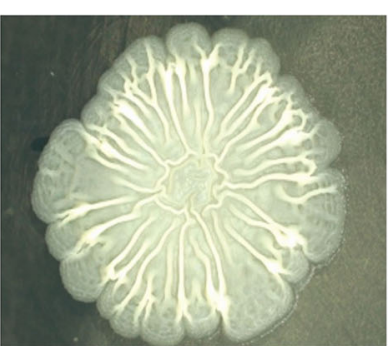

$\triangle a b r B$

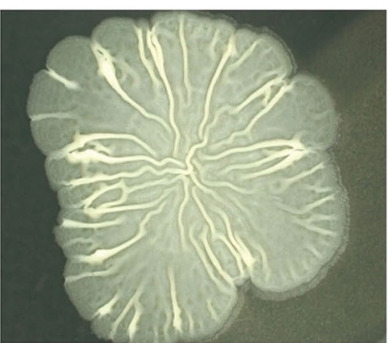

$\triangle s p o V G \Delta a b r B$

Fig. 6 The colonial morphology of B. cereus $0-9$ and its mutants. Tested strains were seeded on NA plates and incubated at $30^{\circ} \mathrm{C}$ for 2 to 3 days. And then, took photos by stereomicroscope. (1) B. cereus 0-9; (2) $\triangle$ spoVG; (3) $\triangle a b r B ;$ (4) $\triangle \operatorname{spoVG} \triangle a b r B$; (5) $\Delta \sin R$; (6) $\Delta \operatorname{spoVG} \Delta \sin R$ 
the same colony characteristics as the single mutant $\triangle a b r B$. For the same reason, it is possible that SpoVG is also located upstream of $a b r B$ and may also regulate the expression of this gene.

\section{SpoVG involves in regulating expression of genes related to biofilm formation of $B$. cereus 0-9}

The sip $W$ and calY are two genes closely related to biofilm formation of B. cereus 0-9 [9]. To confirm the function of SpoVG protein in the biofilm formation process of $B$. cereus $0-9$, the transcription levels of sipW and calY were measured by qRT-PCR, and the results were shown in Table S3. When cultured statically for 3 days in MSgg medium, transcription levels of sipW and calY in the $\Delta$ spoVG mutant were downregulated, compared with levels in wild-type B. cereus 0-9. Transcription of sip $W$ was decreased 19.97-fold and calY was decreased 5.21-fold. These data were consistent with the observed decline of $\triangle$ spoVG biofilm production and indicated that deletion of spoVG gene blocked biofilm formation by suppressing expression of sip $W$ and calY genes, two positive regulators of biofilm formation. This provides additional confirmation for that SpoVG is an important factor controlling biofilm formation of B. cereus 0-9.

To further determine the role of SpoVG in regulating biofilm formation of B. cereus 0-9, several transcriptional fusion strains with PsipW-GFP and PcalYGFP labelling were constructed; details of the constructed strains are shown in Table 1. These transcriptional fusion strains were cultured in MSgg medium, and at different times, samples were taken. Fluorescence intensity was measured and the results are shown in Fig. 7. The GFP intensity of $\Delta$ spoVG (with PsipW-GFP) declined significantly $(P<0.01)$ comparing with wild-type B. cereus 0-9 (with PsipWGFP) after culturing over $12 \mathrm{~h}$ (Fig. 7, A). Similarly, the GFP intensity of $\triangle$ spoVG (with PcalY-GFP) was significantly $(P<0.01)$ difference from that of wild-
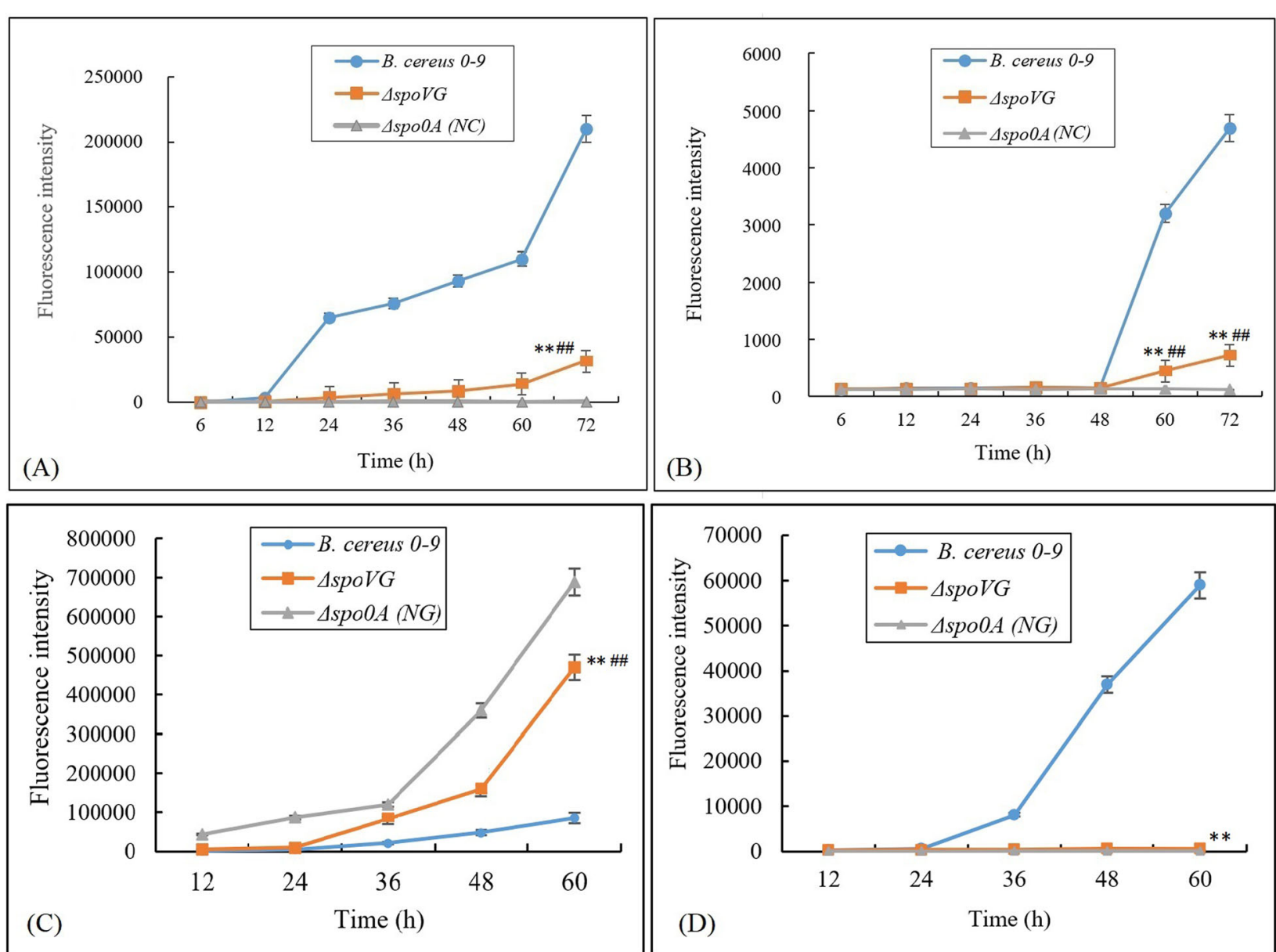

Fig. 7 The fluorescence intensity of transcription fusion strains. B. cereus 0-9 and $\Delta$ spoVG harbouring PsipW-GFP (A), PcalY-GFP (B), PabrB-GFP (C) and Psinl-GFP (D), respectively, were constructed in this experiment. The transcription fusion strains of $\triangle$ spoOA harbouring PsipW-GFP, PcalY-GFP, PabrB-GFP and Psinl-GFP, respectively, was used for negative control (NC). " "**" means $P<0.01$ compared with the data of B. cereus $0-9$ group, "\#\#" means $P<0.01$ compared with the NC group 
type B. cereus 0-9 (with PcalY-GFP) after culturing for $60 \mathrm{~h}$ (Fig. 7, B). This suggested that deletion of spoVG inhibited transcription of sipW and calY.

Fluorescence quantitative PCR and transcriptional fusion technology were adopted to study the transcription levels of $a b r B$ and $\sin I$ in the $\Delta$ spoVG mutant. The transcription level of $a b r B$ increased 11.55fold, while that of $\sin R$ increased 17.39-fold in the $\triangle$ spoVG mutant compared with the wild-type strain of $B$. cereus $0-9$. This indicated that SpoVG negatively regulates transcription of $a b r B$ and $\sin R$, but positively regulates transcription of $\sin I$. Two additional transcriptional fusion vectors, PabrB-GFP and PsinI-GFP, were constructed and inserted into the genomes of $B$. cereus $0-9$ and the $\Delta$ spoVG mutant, respectively, to construct four transcriptional fusion strains, designated as wild-type $B$. cereus $0-9$ with PabrB-GFP, wild-type $B$. cereus 0-9 with PsinI-GFP, $\Delta$ spoVG with PabrB-GFP, and $\Delta$ spoVG with PsinIGFP, and their fluorescence intensity were measured. The expression level of PabrB-GFP in $\Delta s p o V G \mathrm{mu}-$ tant was significant $(P<0.01)$ difference from that in wild B. cereus 0-9 after culturing for $12 \mathrm{~h}$ (Fig. 7, C). However, the expression level of PsinI-GFP in $\Delta$ spoVG mutant decreased significantly $(P<0.01)$ compared with that of wild-type B. cereus 0-9 after culturing for $36 \mathrm{~h}$ (Fig. 7, D). These results indicate that SpoVG participates in the regulation of biofilm formation through both the AbrB system and the SinI/R system.

\section{SpoVG affects $B$. cereus $0-9$ biofilm formation via positively regulating the transcription of SpoOA}

To determine whether SpoVG influenced biofilm formation of B. cereus 0-9 via Spo0A, a series of mutant strains were constructed and used to evaluate the regulatory relationship between SpoVG and Spo0A. The colonial morphology of these mutants observed under stereomicroscope were shown in Fig. 8, and their overall appearance under the digital camera is shown in Fig. S2. Colonies of $\triangle \mathrm{spoVG}, \triangle \mathrm{s} p o 0 A$, and $\triangle \mathrm{spoVG} \Delta \mathrm{spoOA}$ were very smooth and had completely lost the folds on their surface (Fig. 8), which was markedly different from the colony morphology of wild-type $B$. cereus $0-9$. Therefore, these mutants cannot form normal biofilm on the NA surface. The colony morphology of $\triangle$ spoVG $\triangle s p o 0 A$ / spoOA restored some wrinkle biofilm although its surface is not as densely wrinkled as wild-type $B$. cereus $0-9$. However, $\triangle$ spoVG $\triangle \mathrm{spoOA} /$ spoVG mutant could not restore the biofilm-formation, though the spoVG gene was complemented. Thus, we speculated that SpoVG is located upstream of Spo0A in the regulation of biofilm formation and development. But it is very interesting that the colony morphology of $\triangle$ spoVG $\triangle$ spoOA/spoOA was not consistent with that of $\triangle s p o V G$. We further determined the spoOA-overexpressing mutant of $\triangle$ spoVG/

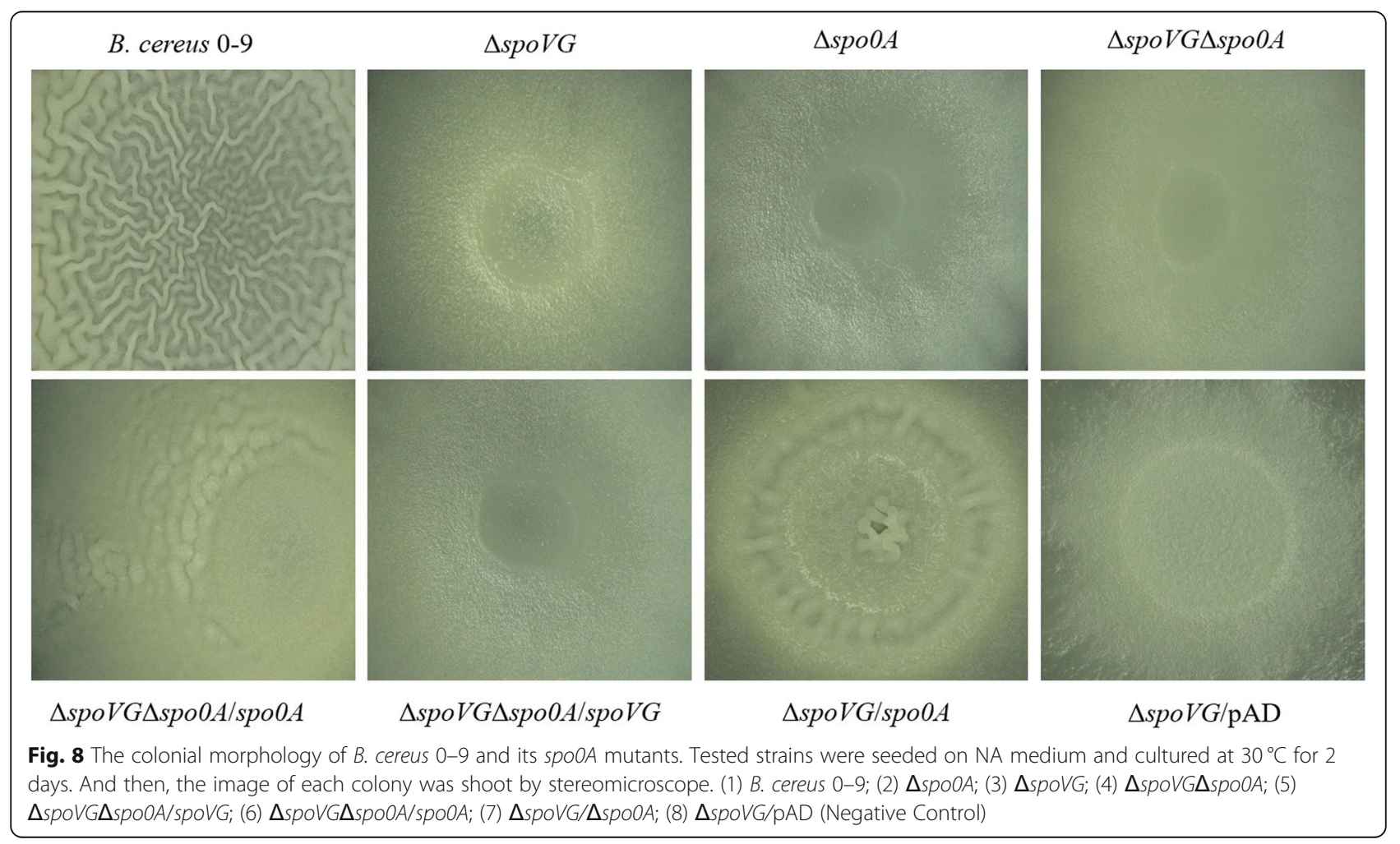


spo0A. As a result, if spoOA was overexpressed in the $\Delta$ spoVG strain, the wrinkled biofilm recovered (Fig. 8). This indicated that the absence of SpoVG can depress the transcription of Spo0A, and consequently the regulatory systems of biofilm formation cannot be activated. Together these results demonstrate that SpoVG is located upstream of Spo0A, and SpoVG is likely to participate in regulating biofilm formation of B. cereus 0-9 through Spo0A.

To further study whether SpoVG can regulate Spo0A, we determined the transcription level of $s p o 0 A$ in the $\triangle$ spoVG mutants by qRT-PCR, and showed the results in Table S3. Compared to wild-type B. cereus $0-9$, the transcription level of spoOA decreased 12.82-fold in the $\triangle$ spoVG mutants. Deletion of spoVG gene inhibited the transcription of spoOA. This indicates that SpoVG has a regulatory effect on Spo0A. The fluorescence assay of transcriptional fusion strains of wild-type $B$. cereus $0-9$ (with Pspo0A-GFP) and $\triangle$ spoVG (with Pspo0A-GFP) was carried out, and the results (Fig. 9) also showed that spoVG gene deletion can inhibited the activity of the promotor of spoOA. As previously mentioned, Spo0A can regulate biofilm formation through $\mathrm{AbrB}$ and $\mathrm{SinI} / \mathrm{R}$ system. For another, we determined the sporulation stage in the MSgg medium which is beneficial to stimulate biofilm formation, and found that $\triangle$ spoVG did not initiate the asymmetric stage of sporulation during the first $36 \mathrm{~h}$ of culture, but the wild-type $B$. cereus $0-9$ did (Fig. S3 and S4). This result is consistent with the inhibition of the transcription level of Spo0A in the $\Delta$ spoVG mutant. Therefore, we concluded that SpoVG can regulate $B$. cereus $0-9$ biofilm formation through Spo0A.

\section{Discussion}

SpoVG, originally identified in B. subtilis as a factor involved in sporulation, is a discrete type of DNA-binding protein commonly found in bacteria [23]. It is broadly conserved, especially among Gram-positive bacteria such as B. subtilis, B. anthracis, B. cereus, Listeria monocytogenes, S. aureus, and Clostridium [4, 29]. SpoVG could alter asymmetric cell division, decreased hemolysin production, and regulated sporulation phenotypes in $B$. subtilis [3]. The homolog of SpoVG in the non-sporulating species $L$. monocytogenes and $S$. aureus was shown to be a modulator of virulence factor synthesis and antibiotic resistance, which may help the organisms coordinate environmental growth and survival [30]. These reports all indicate that SpoVG is a crucial factor for the environmental adaptation of bacteria. B. cereus 0-9, a Grampositive, endospore-forming bacterium isolated from healthy wheat roots by our laboratory, has biological control capacity against several soil-borne plant diseases of wheat. A SpoVG protein (Protein ID: QEF14933.1) encoded by the spoVG gene is present in the genome of B. cereus 0-9 (GenBank: CP042874.1). The current study aimed to determine whether SpoVG plays an important role in the environmental adaptation of B. cereus $0-9$.

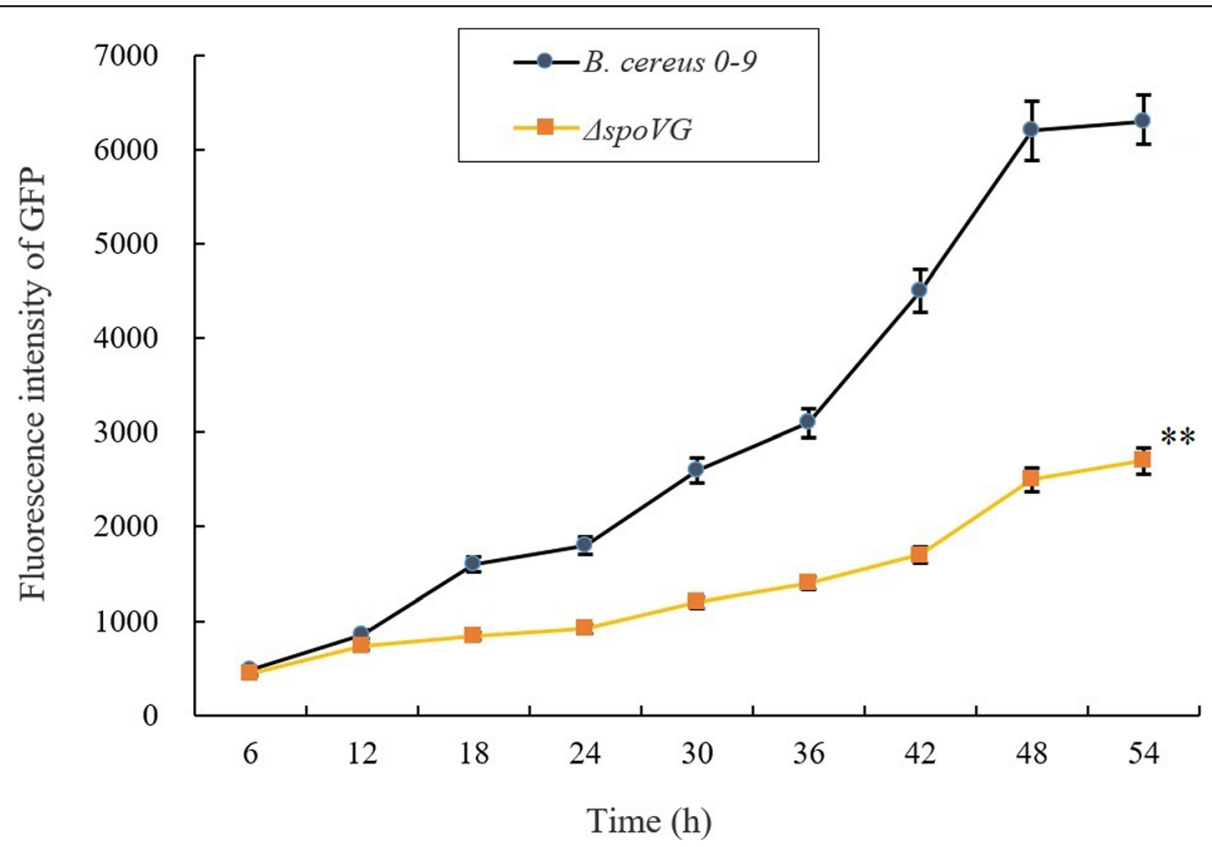

Fig. 9 The fluorescence intensity of the transcription fusion strains. Wild B. cereus 0-9 with Pspo0A-GFP and $\triangle$ spoVG with Pspo0A-GFP were stationarily cultured in MSgg medium at $30^{\circ} \mathrm{C}$. The fluorescence intensity of the tested strains was measured every $6 \mathrm{~h}$. " ${ }^{\prime * * \prime}$ means $P<0.01$ compared with the data of B. cereus 0-9 group 
Despite the discovery of numerous phenotypes of spoVG mutants, the function of the SpoVG protein in bacteria has remained unclear. Studies on SpoVG have predominantly focused on B. subtilis, Bacillus thuringiensis, and S. aureus. In B. subtilis, SpoVG is mainly involved in stage $\mathrm{V}$ of sporulation, that is the formation of the outer membrane of the spore (spore-coat); it negatively regulates asymmetric septum formation and positively regulates cortex formation [3, 4]. SpoVG and SpoIIB jointly determine the sporulation of $B$. subtilis, but single mutants of spoIIB or spoVG in $B$. subtilis showed only minor effects on sporulation [3,31]. Confocal microscopy of $B$. anthracis demonstrated that a $\Delta$ spoVG mutant could not form an asymmetric septum, which is the first morphological change observed during sporulation [4]. SpoVG is highly conserved between $B$. anthracis and B. subtilis, but the effects of the $\Delta$ spoVG mutation on sporulation differ between these two species. Furthermore, the homolog of SpoVG in the nonsporulating species $S$. aureus affected transcription of a number of virulence factors [21, 22]. In the current study, B. cereus $0-9$ could not form mature spores in the absence of SpoVG (as demonstrated in Fig. 1). The asymmetry diaphragm (Septation, phase II) and endocytosis (Engulfment, phase III) of the sporulation process were not affected, but the stage of cortex formation (Cortex/coat, phase IV and V) was affected (Fig. 2). Initial expression of the gene exs $Y$, encoding a major basal layer structural protein (ExsY), occurs when sporulation enters stage $\mathrm{V}$. In the transcriptional fusion assays, the activity of the $\operatorname{exs} Y$ promoter in the transcriptional fusion strain of $\triangle$ spoVG with PexsY-GUS labeling was decreased (in contrast to wild-type strain), meaning the ability of the $\operatorname{Pexs} Y$ promoter was decreased after deletion of the spoVG gene. But the activity of spoIIR promoter in the transcriptional fusion strain of $\triangle$ spoVG (with PspoIIR-GUS) labeling showed no significant $(P>$ $0.05)$ difference with wild-type strain. Therefore, we concluded that SpoVG plays a key role in the sporulation stage $\mathrm{V}$ of $B$. cereus $0-9$.

The SpoVG of B. cereus 0-9 shared $90.72 \%$ homology with that of B. subtilis (SpoVG 168 ), and $100 \%$ sequence identity to SpoVG protein of $B$. anthracis $\left(\mathrm{SpoVG}_{\mathrm{a}}\right)$. However, the function of SpoVG in regulating sporulation of $B$. cereus $0-9$ is quite accordance to that in $B$. subtilis, but different from that in B. anthracis. This difference is probably due to the different metabolic regulation mechanisms of sporulation between different strains. Although both $B$. cereus and B. anthracis belong to the Bacillus cereus group and have similar genomic structures, they carry different plasmids, and their properties differ due to plasmid content or gene expression associated with key regulatory genes [32]. In B. anthracis, there are two virulence plasmid pXO1 (182 kb) and
pXO2 (95 kb), which carries the structural gene of toxin protein and the biosynthetic gene of capsular formation, respectively. In $B$. cereus $0-9$, there were two plasmids with the size of $514 \mathrm{~kb}$ (Accession CP042875) and 14.6 $\mathrm{kb}$ (Accession CP042876), and the former has three Rap-Phr quorum sensing systems. Which may be expressed under specific conditions to regulate the phosphorylation of SpoOF in the phosphoric acid transfer system, thus affecting the phosphorylation degree of the main regulatory factor, SpoOA, and affecting spore formation. This regulatory mechanism is similar to that of B. subtilis, but not B. anthracis. Although the SpoVG protein of B. cereus $0-9$ was $100 \%$ homologous to $\mathrm{SpoVG}_{\mathrm{a}}$, the regulation mechanism of sporulation is different. Therefore, the sporulation of $\triangle$ spoVG mutant of $B$. cereus 0-9 stayed on the stage $\mathrm{V}$, and that of $B$. anthracis was blocked before asymmetric septum formation.

Sporulation is usually initiated in harsh conditions, such as nutritional deficiency, high temperatures, and the presence of sanitizer, that make it impossible for bacteria to survive. In the absence of harsh conditions, spore-forming bacteria often combat adverse environmental factors through swarming, synthesis of various extracellular degrading enzymes, and other stressresistance mechanisms, such as biofilms. Since SpoVG is important in the environmental fitness of bacteria, it is likely to have other functions in addition to regulating sporulation in B. cereus $0-9$.

B. cereus 0-9 has high diversity in lifestyles and ecological niches. It can produce various biofilms that differ in their architecture and mechanism of formation, possibly reflecting an adaptation to varied environments [2]. Biofilm production was investigated and the biofilm formation ability of $B$. cereus $0-9 \Delta$ spoVG was markedly decreased compared with that of wild-type B. cereus 09; this phenotype was restored when the spoVG gene was provided in trans. Therefore, it was concluded that SpoVG is closely related to biofilm formation of $B$. cereus $0-9$. However, the mechanism as to how spoVG regulated biofilm formation was unclear until now. Burke and Portnoya [33] reported that SpoVG was a conserved RNA-binding protein that regulates motility and carbohydrate metabolism, and may positively regulate biofilm formation genes in L. monocytogenes, but no supporting evidence was provided. In the $B$. cereus group, regulation of biofilm formation by SpoVG has not previously been reported. It was therefore surprising in the current study that deletion of spoVG not only caused spore production inhibition of B. cereus $0-9$ in MG medium, but also affected biofilm formation of the strain in stationary culture of MSgg medium. Sporulation and biofilm formation of $B$. cereus have been intensively studied, but there is a lack of information on their 
possible correlation. Therefore, elucidation of the mechanism of SpoVG in regulating biofilm formation, and exploration of possible synergy between biofilm and sporulation may enhance understanding of environmental adaptation of bacteria.

Biofilm formation requires a complex regulatory pathway that coordinates gene expression $[7,8]$. The $\mathrm{SinI} / \mathrm{R}$ system is the most described system for regulating the formation of biofilm in B. cereus [11]. In $B$. subtilis, the proteins TapA, TasA, and SipW are required for biofilm formation [34, 35]. Transcription of the $\operatorname{tap} A$-sip $W$-tas $A$ operon is repressed by $\operatorname{SinR}$, a DNA-binding protein $[10,13]$. In $B$. cereus, CalY has been described as a cell-surface, membrane-bound zinc metallopeptidase that is active against casein, plasminogen, actin, collagen, or fibrinogen, which are closely related to biofilm formation [34-37]. Based on our previous reports, the SinI/R system and CalY protein exist in B. cereus $0-9$ and could regulate biofilm formation [25]. The transcription level of sip $W$ and calY genes were determined by RT-qPCR in the current study. Both sipW and calY were downregulated when the spoVG gene was deleted. Furthermore, construction and analysis of the GFP transcriptional fusion strains (Table 1) demonstrated that activity levels of the promoters of sip $W$ and calY decreased when spoVG was deleted. These results indicated that SpoVG could regulate biofilm formation of $B$. cereus 0-9 through the SinI/R system. Moreover, colony morphology of $\Delta$ spoVG $\Delta \sin R$ were identical to that of the $\Delta \sin R$ mutant, which confirmed the findings of the transcriptional-fusion assays, and indicated that spoVG is located upstream of $\sin R$. Together, these data suggest that SpoVG regulates biofilm formation of $B$. cereus 0-9 through the SinR system.

According to previous reports, B. subtilis forms biofilms in a process that is negatively controlled by the transcription factor AbrB [38]. To determine whether the same process occurred in B. cereus, GFP transcription fusion strains were constructed and their green fluorescence intensity was measured during the culturing process. Activity of the $a b r B$ promoter in $\triangle s p o V G$ mutant was higher than that in wild strain after culturing for $12 \mathrm{~h}$. Further RT-qPCR results revealed that $a b r B$ gene expression in the $\triangle$ spoVG strain was upregulated 11.55-fold compared with that of the wild-type B. cereus $0-9$. Moreover, the colony fold phenotype of the $\triangle a b r B$ strain formed on the plate surface was distinct from that of wild-type $B$. cereus $0-9$, while the colony surface folds of the double mutant $\triangle$ spoVG $\triangle a b r B$ were identical to those of the single deletion mutant $\triangle a b r B$ (Fig. 6). This indicated that spoVG is located upstream of $a b r B$, and that SpoVG participates in the regulation of biofilm formation through negative regulation of $\mathrm{AbrB}$ in $B$. cereus
$0-9$. Meanwhile, the transcription level of $\sin R$ was upregulated 17.39-fold in $\triangle$ spoVG mutant compared with that of wild-type B. cereus $0-9$. SinR is a key biofilm master repressor, and $\operatorname{SinI}$ is a small protein antagonist of $\operatorname{Sin} R$ [39]. The genes $\sin I$ and $\sin R$ exist in tandem in the genome of $B$. cereus and share the same promoter located upstream of $\sin I$ [16]. The upregulated transcription level of $\sin R$ could de-repress the biofilm formation [10]. These data from the literature, combined with those from the current study, further confirm that SpoVG is involved in regulating biofilm formation of B. cereus 0-9 through the AbrB and SinI/R systems.

Spo0A, a member of the response regulator family of transcriptional regulators, can be activated by multiple histidine kinases, sensing environmental and physiological signals, to regulate the biofilm formation of $B$. subtilis and B. cereus [16, 38, 40] (Fig. 8). Spo0A also activates biofilm formation by increasing production of SinI [40]. The role of Spo0A in biofilm formation is to negatively regulate $\mathrm{AbrB}$, which is another transcription factor that negatively regulates biofilm formation of $B$. subtilis [38]. To further explore the systemic mechanism of the involvement of SpoVG in regulation of biofilm formation, the direct regulatory relationship between SpoVG and Spo0A was investigated. The biofilm fold of $\triangle$ spoVG $\triangle$ spoOA strain was eliminated, but it could be recovered by complementation of spoOA gene alone, such as that of $\triangle$ spoVG $\triangle$ spoOA/SpoOA mutant. However, the biofilm was not restored by complementation of spoVG gene, such as $\Delta$ spoVG $\Delta$ spoOA/SpoVG (Fig. 8). This proved that spoVG was located upstream of spoOA. Based on the process of biofilm development in B. cereus $0-9$, spoVG is located upstream of spoOA and regulates the expression of Spo0A. Furthermore, it is surprising to observe that the biofilm development of $\triangle$ spoVG $\Delta$ spoOA/spoOA was not identified with $\triangle$ spoVG. If spoOA is overexpressed in $\triangle s p o V G$, the biofilm recovers, therefore, colony morphology of $\triangle$ spoVG/spoOA is consistent with that of $\triangle$ spoVG $\triangle$ spoOA/spoOA mutant. It may due to the overexpression of the reverse complementary vector of pAD-spo0A. These results indicate that SpoVG may be a regulatory factor of Spo0A in the process of $B$. cereus 0-9 biofilm formation. SpoVG has been reported to play a role in the spore-coat formation stage of sporulation, while Spo0A was reported to be involved in the initial stage of spore differentiation, that is, spoVG is located downstream of $s p o O A$ and is regulated by SpoOA $[4,18,38]$. But our conclusion is contrary to these reports. This may be due to different metabolic regulation mechanisms in B. cereus 0-9 under different culture conditions. In germination medium, $\Delta$ spoVG did not form mature spores and can initiated sporulation but stop them in the stage VI. However, the biofilm production of $\Delta$ spoVG was inhibited in the static cultivation of 
MSgg medium. Therefore, we further explored the regulatory relationship between SpoVG and Spo0A in the biofilm-forming condition.

According to the previous report of $\mathrm{Zhu}$ et al. [41], SpoVG is a negative regulator and directly represses the expression of $s a s C$, which could specifically bind to the promoter region of sasC in $S$. aureus. Thus, we examined whether SpoVG affects the transcription of Spo0A. RT-qPCR analysis further demonstrated that the transcription level of $s p o O A$ in the $\triangle$ spoVG strain decreased 12.82-folds compared with that in wild-type $B$. cereus 0 9 in MSgg medium. For another, we constructed the transcriptional fusion strains of $B$. cereus $0-9$ and $\triangle$ spoVG with PspoOA-GFP, respectively, in the biofilmforming condition. The determination of fluorescence intensity shows that spoVG gene deletion inhibited the activity of promoter of Spo0A. These results indicate that the absence of SpoVG reduces the expression level of Spo0A, and consequently the regulating systems of biofilm formation cannot be activated. Therefore, we conclude that SpoVG participates in biofilm formation of $B$. cereus 0-9 may through regulating the transcription level of spoOA.

Spo0A is a well-known transcriptional factor required for early sporulation [42]. Since biofilm formation is also regulated by Spo0A, this indicates that sporulation and biofilm development may be intrinsically linked in Bacillus. Intertwined regulatory pathways between biofilm formation and sporulation have been proposed for $B$. subtilis [8], and could be similar in B. cereus [40]. It is reported that the bacterial cells have the option of taking either the sporulation or biofilm development pathways upon activation of Spo0A [38]. When Spo0A P levels are relatively low during the early stages of starvation, biofilm is formed, but sporulation does while the Spo0A $\sim \mathrm{P}$ levels are high [43]. Thus, we determined the sporulation stage in the biofilm-forming condition, and found that $\triangle$ spoVG did not initiate the asymmetric stage of sporulation during the first $36 \mathrm{~h}$ of culture, but the wildtype B. cereus 0-9 did (Fig. S3 and S4). This indicated that spoVG gene mutation also inhibited the spore differentiation of $B$. cereus 0-9 under this culture condition. In $B$. thuringiensis, three distinct cell types controlled by quorum-sensing regulators have been identified: PlcR-controlled virulent cells, NprRdependent necrotrophic cells, and cells committed to sporulation [44]. In the developmental stages of B. thuringiensis, virulence, necrotrophism, and sporulation processes are three distinct physiological pathways controlled by Rap phosphatases and Spo0A-P [44]. In another related development, gradual accumulation of Spo0A $\sim \mathrm{P}$ is essential for the proper temporal order of the Spo0A regulon expression, and that reduction in sporulation efficiency results from the reversal of that order (Monika et al., 2013). It is thus possible that the phosphorylation level of Spo0A $\sim \mathrm{P}$ is a key regulatory mechanism that controls the initiation of biofilm formation or sporulation by B. cereus $0-9$. This mechanism is so interesting that it will attract us to explore it in our further work.

\section{Conclusions}

As a biocontrol bacterium derived from soil, B. cereus 0-9 has evolved various environmental adaptation mechanisms, such as swarming motility, biofilm formation, spore differentiation, etc. SpoVG is a regulator that is broadly conserved, especially among many Grampositive bacteria, and may help the organisms coordinate environmental growth and to survival. SpoVG regulates stage $\mathrm{V}$ of sporulation in $B$. cereus $0-9$, which is identical to the process in B. subtilis, but differs to that of $B$. anthracis. Since SpoVG plays a crucial role in environmental fitness of bacteria, it is likely to have other functions in addition to regulating sporulation in B. cereus $0-9$. Biofilm production of $\triangle$ spoVG mutants were therefore studied and SpoVG was demonstrated to be involved in regulating biofilm formation of B. cereus 0-9. Transcriptional fusion strains, double gene mutant strains and their complemented mutants were used to explore the mechanism of SpoVG regulation of biofilm formation in B. cereus $0-9$, and the results consistently showed that SpoVG regulated biofilm formation of $B$. cereus 0-9 through both the $A b r B$ and $\operatorname{SinI} / R$ systems. Analysis of the direct regulatory relationship between SpoVG and Spo0A led to the conclusion that SpoVG is located upstream of Spo0A and participates in regulation of biofilm formation of B. cereus $0-9$ through regulating the transcription level of spoOA. This study demonstrates that SpoVG is an important regulator of Spo0A, which is crucial for both sporulation and biofilm formation of B. cereus 0-9. SpoVG is therefore important for the environmental adaptability of $B$. cereus. The findings of this study enhance understanding of bacterial biofilms and may facilitate the development new biological control strategies utilizing bacteria.

\section{Abbreviations \\ MG: Modified germination; NA: Nutrient agar; MSgg: Minimal salts glycerol glautamate; MU: 4-methylumbelliferyl; MUG: 4-methylumbelliferyl beta-D- glucuronide dihydrate; GFP: Green fluorescent protein; qRT-PCR: Real-time quantitative PCR; CFU: Colony-forming units}

\section{Supplementary Information}

The online version contains supplementary material available at https://doi. org/10.1186/s12866-021-02239-6.

Additional file 1: Table S1. Primers used in this text. Table S2. The spore yield rate of experiment strains with different culture time. Table S3. Gene expression level of sipW, calY, $\sin R$ and $a b r B$ in B. cereus $0-9$ and $\triangle$ spoVG determined by qRT-PCR. Figure S1. The GUS 
enzymatic activities of wild $B$. cereus $0-9$ and $\Delta$ spoVG mutant at MG medium under different culturing times. One $U$ of GUS was defined as the change of fluorescence intensity per unit of protein in per hour. Each bar represents mean and standard deviations of the mean of all the 3 measurements. Figure S2. The colonial morphology of B. cereus 0-9 and its spoOA mutants. Tested strains were seeded on NA medium and cultured at $30^{\circ} \mathrm{C}$ for 2 days. And then, the image of each colony was shoot by digital camera. (1) B. cereus 0-9; (2) $\Delta$ spoOA; (3) $\Delta$ spoVG; (4) $\Delta$ spoVG $\Delta$ spoOA; (5) $\Delta$ spoVG $\triangle$ spoOA/spoVG; (6) $\triangle$ spoVG $\Delta$ spoOA/spoOA; (7) $\Delta$ spoVG/ $\triangle$ spoOA; (8) $\triangle$ spoVG/pAD (Negative Control). Figure S3. Sporulation of $B$. cereus $0-9$ when it was cultured in MSgg medium at $30^{\circ} \mathrm{C}, 220 \mathrm{rpm}$ for $24 \mathrm{~h}, 36 \mathrm{~h}, 42 \mathrm{~h}, 48 \mathrm{~h}$ and $56 \mathrm{~h}$, respectively, and stained with two fluorescent dyes. And then, observed under a fluorescence microscope. For the membrane, only the red fluorescence signal of FM4-64 was collected, that is, the cell membrane and spore-coat were stained red; For the cell nucleus, only the blue signal of DAPI is collected, that is, the nuclear DNA is stained blue; And the merge images of the membrane and nuclear DNA showed the overall perspective. Figure S4. Sporulation of $\Delta$ spoVG mutant when it was cultured in MSgg medium at $30^{\circ} \mathrm{C}, 220 \mathrm{rpm}$ for $24 \mathrm{~h}$, $36 \mathrm{~h}, 42 \mathrm{~h}$ and $48 \mathrm{~h}$, respectively, and stained with two fluorescent dyes. And then, observed under a fluorescence microscope. For the membrane, only the red fluorescence signal of FM4-64 was collected, that is, the cell membrane and spore-coat were stained red; For the cell nucleus, only the blue signal of DAPI is collected, that is, the nuclear DNA is stained blue; And the merge images of the membrane and nuclear DNA showed the overall perspective.

\section{Acknowledgments}

The authors would like to thank the Microbiology Bioengineering Experimental Center of Henan University for assistance with figures and feedback on an early manuscript draft.

\section{Authors' contributions}

$\mathrm{QH}$ carried out the experiment of the mechanism of biofilm formation and determined the sporulation process and spore yields of test strains, constructed all the transcriptional fusion strains and helped collecting the Figs. ZZ helped to design some experiments and carried out the experiment of quantitative detection of biofilms, and done a lot of photographing and sorting work of experimental pictures. QL constructed the expression vectors and gene knockout strains, and has done a lot of work in pioneering studies such as the phenotypic determination of mutant strains and gene expression level. FL carried out the experiment of determination of qRT-PCR and collected test data. $Y L$ performed the data analyses and helped the experiment going. JZ drafted the manuscript and revised the paper, and helped design the experiment. GW led the relevant project and designed the experiment. All authors read and approved the final manuscript.

\section{Funding}

This study was funded by two National Nature Science Foundation of China (Number: 31572047 and 31701831) and Science and Technology Research and Development Project of Henan Province (212300410331 and 212102310910). National Nature Science Foundation of China mainly funded the experimental implementation, data collection, analysis and manuscript writing of this study. Technology Research and Development Project of Henan Province mainly funded the experimental design and experimental materials.

\section{Availability of data and materials}

Data on the genomes of $B$. cereus 0-9 have been submitted to the Gene Bank of NCBI which is open, and its GenBank ID is CP042874.1. The website is as follow: https://Www.ncbinlm.nih.gov/nuccore/CP042874.1/ And all the other data generated during this study are included in this published article [and its supplementary information files.

\section{Declarations}

Ethics approval and consent to participate Not applicable.

\section{Consent for publication}

Not applicable.

\section{Competing interests}

The authors declare that they have no competing interests.

\section{Author details}

${ }^{1}$ Institute of Microbial Engineering, Laboratory of Bioresource and Applied Microbiology, School of Life Sciences, Henan University, Jinming Street, Kaifeng 475004, China. ${ }^{2}$ Engineering Research Center for Applied Microbiology of Henan Province, Kaifeng 475004, China. ${ }^{3}$ School of Pharmaceutical, Henan Univeristy, Kaifeng 475004, China.

Received: 18 April 2021 Accepted: 20 May 2021

Published online: 08 June 2021

\section{References}

1. Moons $\mathrm{P}$, Chris M, Abram A. Bacterial interactions in biofilms. Crit Rev Microbiol. 2009;35:157-68.

2. Majed R, Faille C, Kallassy M, Gohar M. Bacillus cereus biofilms-same, only different. Front Microbiol. 2016;7:1054.

3. Matsuno K, Sonenshein AL. Role of SpoVG in asymmetric septation in Bacillus subtilis. J Bacteriol. 1999;181:3392-401.

4. Chen M, Lyu Y, Feng E, Zhu L, Pan C, Wang D, et al. SpoVG is necessary for sporulation in Bacillus anthracis. Microorganisms. 2020;8:548.

5. Boone TJ, Mallozzi M, Nelson A, Thompson B, Khemmani M, Lehmann D, et al. Coordinated assembly of the Bacillus anthracis coat and exosporium during bacterial spore outer layer formation. Mol Biol Physiol. 2018;9: e01166-18.

6. Hagan AK, Plotnick YM, Dingle RE, Mendel ZI, Cendrowski SR, Sherman DH, et al. Petrobactin protects against oxidative stress and enhances sporulation efficiency in Bacillus anthracis Sterne. Mol Biol Physiol. 2018;9:e02079-18.

7. Romero D, Vlamakis H, Losick R, Kolter R. Functional analysis of the accessory protein TapA in Bacillus subtilis amyloid fiber assembly. J Bacteriol. 2014:196:1505-13.

8. Vishnoi M, Narula J, Devi SN, Dao HA, Igoshin OA, Fujita M. Triggering sporulation in Bacillus subtilis with artificial two-component systems reveals the importance of proper Spo0A activation dynamics. Mol Micro. 2013;115: $181-94$

9. Zhang J, Wang H, Xie T, Huang Q, Xiong X, Liu Q, et al. The YmdB protein regulates biofilm formation dependent on the repressor $\operatorname{Sin} R$ in Bacillus cereus 0-9. World J Microbiol Biotechnol. 2020;36:165.

10. Diethmaier C, Newman JA, Akos TK, Kaever V, Stülke J. The YmdB phosphodiesterase is a global regulator of late adaptive responses in Bacillus subtilis. J Bacteriol. 2014;196:265-75.

11. Newman JA, Rodrigues C, Lewis RJ. Molecular basis of the activity of SinR protein, the master regulator of biofilm formation in Bacillus subtilis. J Biol Chem. 2013;288:10766-78.

12. Branda SS, Gonzalez-Pastor JE, Ben-Yehuda S, Losick R, Kolter R. Fruiting body formation by Bacillus subtilis. Proc Natl Acad Sci U S A. 2001;98:11621-6.

13. Kearns DB, Chu F, Branda SS, Kolter R, Losick R. A master regulator for biofilm formation by Bacillus subtilis. Mol Microbiol. 2005;55:739-49.

14. Lewis RJ, Brannigan JA, Smith I, Wilkinson AJ. Crystallisation of the Bacillus subtilis sporulation inhibitor $\operatorname{SinR}$, complexed with its antagonist. Sinl FEBS Lett. 1996:378:98-100.

15. Lewis RJ, Brannigan JA, Offen WA, Smith I, Wilkinson AJ. An evolutionary link between sporulation and prophage induction in the structure of a repressor: anti-repressor complex. J Mol Biol. 1998;283:907-12.

16. Xu S, Yang N, Zheng S, Yan F, Jiang C, Yu Y, et al. The spooA-sinl-sinR regulatory circuit plays an essential role in biofilm formation, nematicidal activities, and plant protection in Bacillus cereus AR156. MPMI. 2017. https://doi.org/10.1094/MPMI-02-17-0042-R.

17. Hamon MA, Stanley NR, Britton RA, Grossman AD, Lazazzera BA. Identification of AbrB-regulated genes involved in biofilm formation by Bacillus subtilis. Mol Microbiol. 2004;52:847-60.

18. Rosenbluh A, Banner CDB, Losick R, Fitz-James PC. Identification of a new developmental locus in Bacillus subtilis by construction of a deletion mutation in a cloned gene under sporulation control. J Bacteriol. 1981;148: $341-51$. 
19. Liu X, Zhang S, Sun B. SpoVG regulates cell wall metabolism and oxacillin resistance in methicillin-resistant Staphylococcus aureus strain N315. Antimicrob Agents Chemother. 2016;60:3455-61.

20. Savage $C R$, Jutras BL, Bestor A, Tilly K, Rosa PA, Tourand Y, et al. Borrelia burgdorferi SpoVG DNA- and RNA-binding protein modulates the physiology of the Lyme disease spirochete. J Bacteriol. 2018;200:e00033-18.

21. Meier S, Goerke C, Woiz C, Seidl K, Homerova D, Schulthess B, et al. Sigma(B) and the sigma(B)-dependent arlRS and yabJ-spoVG loci affect capsule formation in Staphylococcus aureus. Infect Immun. 2007;75:4562-71.

22. Schulthess B, Bloes DA, Francois P, Girard M, Schrenzel J, Bischoff $M$, et al. The sigma(B)-dependent yabJ-spoVG operon is involved in the regulation of extracellular nuclease, lipase, and protease expression in Staphylococcus aureus. J Bacteriol. 2011:193:4954-62.

23. Jutras BL, Chenail AM, Rowland CL, Dustin C, Clarke MM, Tomasz B, et al. Eubacterial SpoVG homologs constitute a new family of site-specific DNAbinding proteins. PLoS One. 2013;8:e66683.

24. Zhang J, Wang H, Huang Q, Zhang Y, Zhao L, Liu F, et al. Four superoxide dismutases of Bacillus cereus 0-9 are non-redundant and perform different functions in diverse living conditions. World J Microbiol Biotechnol. 2020;36: $1-12$.

25. Zhang J, Li M, Zhang Y, Sang L, Liu Q, Zhao L, et al. GapB is involved in biofilm formation dependent on LrgAB but not the Sinl/R system in Bacillus cereus 0-9. Front Microbiol. 2020. https://doi.org/10.3389/fmicb.2020.591926.

26. Xu Y, Chen M, Zhang Y, Wang M, Ying W, Huang Q, et al. The phosphotransferase system gene pts/ in the endophytic bacterium Bacillus cereus is required for biofilm formation, colonization, and biocontrol against wheat sharp eyespot. FEMS Microbio Let. 2014;354(2):142-52.

27. Oktari A, Supriatin Y, Kamal M, Syafrullah H. The bacterial endospore stain on Schaeffer-Fulton using variation of methylene blue solution. J Phys Conf Ser. 2017:812:1-6.

28. Hilbert DW, Piggot PJ. Compartmentalization of gene expression during Bacillus subtilis spore formation. Microbiol mol biol. 2004;68:234-62.

29. Liu J, Prindle A, Humphries J, Gabalda-Sagarra M, Asally M, Lee DD, et al. Metabolic co-dependence gives rise to collective oscillations within biofilms. Cell. 2015;523:550-66.

30. Bischoff M, Brelle S, Minatelli S, Molle V. Stk1-mediated phosphorylation stimulates the DNA-binding properties of the Staphylococcus aureus SpoVG transcriptional factor. Biochem Biophys Res Commun. 2016:473:1223-8.

31. Londono-Vallejo JA, Frehel C, Stragier P. Spoll Q, a forespore-expressed gene required for engulfment in Bacillus subtilis. Mol Microbiol. 1997;24:29-39.

32. Ehling-Schulz M, Koehler TM, Lereclus D. The Bacillus cereus group: Bacillus species with pathogenic potential. Microbiol Spectr. 2019;7(3):1-60.

33. Burke TP, Portnoya DA. SpoVG is a conserved RNA-binding protein that regulates Listeria monocytogenes lysozyme resistance, virulence, and swarming motility. mBio. 2017;7:e00240-16.

34. Candela T, Fagerlund A, Buisson C, Gilois N, Kolstø A, Økstad O, et al. CalY is a major virulence factor and a biofilm matrix protein. Mol Microbiol. 2019; 111:1416-29.

35. Caro-Astorga J, Pérez-García A, De Vicente A, Romero D. A genomic region involved in the formation of adhesin fibers in Bacillus cereus biofilms. Front Microbiol. 2015:5:1-11.

36. Fricke B, Drossler K, Willhardt I, Schierhorn A, Menge S, Rucknagel P. The cell envelope-bound metalloprotease (camelysin) from Bacillus cereus is a possible pathogenic factor. Biochim Biophys Acta. 2001;1537: $132-46$

37. Grass G, Schierhorn A, Sorkau E, Muller H, Rucknagel P, Nies DH, et al. Camelysin is a novel surface metalloproteinase from Bacillus cereus. Infect Immun. 2004;72:219-28.

38. Hamon MA, Lazazzera BA. The sporulation transcription factor Spo0A is required for biofilm development in Bacillus subtilis. Mol Microbiol. 2001;42: 1199-209.

39. Bai U, Mandic-Mulec I, Smith I. Sinl modulates the activity of SinR, a developmental switch protein of Bacillus subtilis, by protein-protein interaction. Genes Dev. 1993;7:139-48.

40. Huang Y, Flint SH, Palmer JS. Bacillus cereus spores and toxins - the potential role of biofilms. Food Microbiol. 2020;90:e103493.

41. Zhu Q, Liu B, Sun B. SpoVG modulates cell aggregation by regulating sasC expression and eDNA. Appl Environ Microbiol. 2020;86:e00591-20.

42. Burbulys $D$, Trach KA, Hoch JA. Initiation of sporulation in B subtilis is controlled by a multicomponent phosphorelay. Cell. 1991;64:545-52.
43. Fujita M, Losick R. Evidence that entry into sporulation in Bacillus subtilis is governed by a gradual increase in the level and activity of the master regulator Spo0A. Genes DevGene Dev. 2005;19:2236-44.

44. Verplaetse E, Slamti L, Gohar M, Lereclus D. Two distinct pathways lead Bacillus thuringiensis to commit to sporulation in biofilm. Res Microbiol. 2017;168:388e393.

\section{Publisher's Note}

Springer Nature remains neutral with regard to jurisdictional claims in published maps and institutional affiliations.
Ready to submit your research? Choose BMC and benefit from:

- fast, convenient online submission

- thorough peer review by experienced researchers in your field

- rapid publication on acceptance

- support for research data, including large and complex data types

- gold Open Access which fosters wider collaboration and increased citations

- maximum visibility for your research: over $100 \mathrm{M}$ website views per year

At BMC, research is always in progress.

Learn more biomedcentral.com/submissions 